\title{
Activity of Tachykinin1-Expressing Pet1 Raphe Neurons Modulates the Respiratory Chemoreflex
}

\author{
Morgan L. Hennessy, ${ }^{1}$ Andrea E. Corcoran, ${ }^{2}$ Rachael D. Brust, ${ }^{1}$ YoonJeung Chang, ${ }^{1}$ Eugene E. Nattie, ${ }^{2}$ \\ and ${ }^{\circ S u s a n}$ M. Dymecki ${ }^{1}$ \\ ${ }^{1}$ Department of Genetics, Harvard Medical School, Boston, Massachusetts 02115, and 2Department of Physiology and Neurobiology, Geisel School of \\ Medicine at Dartmouth, Lebanon, New Hampshire 03756
}

Homeostatic control of breathing, heart rate, and body temperature relies on circuits within the brainstem modulated by the neurotransmitter serotonin (5-HT). Mounting evidence points to specialized neuronal subtypes within the serotonergic neuronal system, borne out in functional studies, for the modulation of distinct facets of homeostasis. Such functional differences, read out at the organismal level, are likely subserved by differences among 5-HT neuron subtypes at the cellular and molecular levels, including differences in the capacity to coexpress other neurotransmitters such as glutamate, GABA, thyrotropin releasing hormone, and substance P encoded by the Tachykinin-1 (Tac1) gene. Here, we characterize in mice a 5-HT neuron subtype identified by expression of Tac1 and the serotonergic transcription factor gene Pet1, referred to as the Tac1-Pet1 neuron subtype. Transgenic cell labeling showed Tac1-Pet1 soma resident largely in the caudal medulla. Chemogenetic [clozapine- $N$-oxide (CNO)-hM4Di] perturbation of Tac1-Pet1 neuron activity blunted the ventilatory response of the respiratory $\mathrm{CO}_{2}$ chemoreflex, which normally augments ventilation in response to hypercapnic acidosis to restore normal $\mathrm{pH}$ and $\mathrm{PCO}_{2}$. Tac1-Pet1 axonal boutons were found localized to brainstem areas implicated in respiratory modulation, with highest density in motor regions. These findings demonstrate that the activity of a Pet 1 neuron subtype with the potential to release both 5-HT and substance $\mathrm{P}$ is necessary for normal respiratory dynamics, perhaps via motor outputs that engage muscles of respiration and maintain airway patency. These Tac1-Pet1 neurons may act downstream of Egr2-Pet1 serotonergic neurons, which were previously established in respiratory chemoreception, but do not innervate respiratory motor nuclei.

Significance Statement

Serotonin (5-HT) neurons modulate physiological processes and behaviors as diverse as body temperature, respiration, aggression, and mood. Using genetic tools, we characterize a 5-HT neuron subtype defined by expression of Tachykinin1 and Pet1 (Tac1-Pet1 neurons), mapping soma localization to the caudal medulla primarily and axonal projections to brainstem motor nuclei most prominently, and, when silenced, observed blunting of the ventilatory response to inhaled $\mathrm{CO}_{2}$. Tac1-Pet1 neurons thus appear distinct from and contrast previously described Egr2-Pet1 neurons, which project primarily to chemosensory integration centers and are themselves chemosensitive.

\section{Introduction}

Serotonin (5-HT)-producing neurons in the lower brainstem are the subject of intense investigation given their importance in

Received July 21, 2016; revised Nov. 30, 2016; accepted Dec. 30, 2016.

Author contributions: M.L.H., A.E.C., R.D.B., E.E.N., and S.M.D. designed research; M.L.H., A.E.C., and Y.J.C. performed research; M.L.H., A.E.C., E.E.N., and S.M.D. analyzed data; M.L.H., E.E.N., and S.M.D. wrote the paper.

This work was supported by the National Institutes of Health (Grant P01 HD036379 to S.M.D, E.E.N., and A.E.C.; National Institute of General Medical Sciences Grants F31NS073276 to R.D.B. and T32GM007753 to M.L.H.; and National Institute of Neurological Disorders and Stroke Grant F31 NS083165- 02 to M.L.H). The content is solely the responsibility of the authors and does not necessarily represent the official views of the National Institute of General Medical Sciences or the National Institutes of Health. We thank B. Rood, B. Okaty, M. Freret, and R. Dosumu-Johnson for discussion; J.J. Mai for technical support; the Harvard Neurobiology Imaging Center for microscopy support; M. Rice for help with figure graphics; and R. Ouillette for experimental assistance.

The authors declare no competing financial interests. life-sustaining modulation of breathing, heart rate, and body temperature. These functions in rodents may be coordinated in part by specialized subsets of 5-HT neurons, distinguishable and perhaps functionally divisible by coexpression of various neurotransmitters. For example, some medullary serotonergic neurons coexpress substance $\mathrm{P}$, thyrotropin-releasing hormone,

A. Corcoran's present address: John Merck Division of Science and Technology, Southern Vermont College, Bennington, VT.

R.D. Brust's present address: Department of Cell Biology, Harvard Medical School, Boston, MA.

Correspondence should be addressed to Susan M. Dymecki, Department of Genetics, Harvard Medical School, 77 Avenue Louis Pasteur, New Research Building, Room 358, Boston, MA 02115. E-mail: dymecki@genetics.med.harvard.edu.

DOI:10.1523/JNEUROSCI.2316-16.2016

Copyright $\odot 2017$ the authors $\quad 0270-6474 / 17 / 371807-13 \$ 15.00 / 0$ 
GABA, met-enkephalin, or leu-enkephalin (Glazer et al., 1981; Kachidian et al., 1991; Pilowsky, 2014). Transcriptional profiling of individual serotonergic neurons supports similar conclusions, revealing molecularly distinct subtypes of 5-HT neurons (Spaethling et al., 2014; Okaty et al., 2015), including subtypes that not only express the gene encoding tryptophan hydroxylase 2 (Tph2) for synthesis of 5-HT, but also neurotransmitter genes such as Tachykinin 1 (Tac1) encoding substance $\mathrm{P}$, or enzyme genes such as Gad1, encoding GAD-67 for GABA synthesis. Tac1expressing Pet1+ neurons, annotated as Tac1-Pet1 neurons, populate the medullary raphe, especially the raphe obscurus (Okaty et al., 2015). Gad1-expressing Pet1 + neurons were identified in a 5-HT neuronal lineage defined by embryonic expression of Egr2 and by arising from rhombomere 5 ( $\mathrm{r} 5)$. These mature r5Egr2-Pet1 neurons have GABAergic and serotonergic features, as suggested by prior anatomical studies (Stamp and Semba, 1995). Differential expression of Tac1, Egr2, and Gad1 by 5-HT neurons or their precursors suggests different functionalities and offers genetic access to subtypes of 5-HT neurons when used as Cre drivers partnered with the Flpe driver transgene Pet1:: Flpe (Jensen et al., 2008) and various intersectional reporter and effector tools (Ray et al., 2011; Brust et al., 2014; Niederkofler et al., 2016). Here, we apply these tools to characterize the function and projections of Tac1-Pet1 neurons.

Studies have linked the control of breathing to 5-HT, substance $\mathrm{P}$, its receptor, the neurokinin-1 receptor (NK1R), and medullary raphe neurons more generally (Gray et al., 2001; Wang et al., 2001; Nattie and Li, 2006; Berner et al., 2007; Hodges and Richerson, 2008; Ptak et al., 2009; Cummings et al., 2011b; Ray et al., 2011). Only recently, though, have tools with sufficient resolving power become available to explore double-positive 5-HT/substance P neurons; that is, Tac1-Pet1 neurons. Prior studies probing raphe obscurus neurons based on anatomical location have implicated these neurons in respiratory control (Peever et al., 2001; Dias et al., 2008; Nuding et al., 2009; Depuy et al., 2011). For example, injection of Cre-dependent virus targeting channelrhodopsin2-mCherry to caudal raphe Pet1-expressing neurons demonstrated stimulation of breathing upon activation and the presence of axon fibers in brainstem motor nuclei and respiratory rhythm generation regions (Depuy et al., 2011). We hypothesized that the 5-HT neuron subtype responsible for this effect is the Tac1-Pet1 subtype because it overlaps anatomically with the 5-HT neurons captured by viral injection and because fibers harboring substance $\mathrm{P}$, in some cases colocalized with 5-HT, map to many of the same targets (Thor and Helke, 1987; Holtman, 1988; Tallaksen-Greene et al., 1993; Nakamura et al., 2006), which themselves are NK1R+.

Our previous studies showed that Egr2-Pet1 neurons are required for a normal increase in ventilation in response to inspired $5 \% \mathrm{CO}_{2}$ via the respiratory chemoreflex, which maintains tissue $\mathrm{PCO}_{2}$ and $\mathrm{pH}$ within physiological range. In addition, r5Egr2Pet1 neurons were found to be intrinsically chemosensitive, increasing firing in response to hypercapnic acidosis, collectively indicating a role as chemoreceptors that facilitate respiratory drive (Brust et al., 2014). Downstream targets include other chemoreceptor centers such as the retrotrapezoid nucleus; however, innervation of respiratory motor nuclei under serotonergic modulation was absent (Brust et al., 2014). The latter, we posit, may be provided by Tac1-Pet1 neurons. To explore this, we partnered a Tac1::IRES-cre driver (Harris et al., 2014) with Pet1::Flpe (Jensen et al., 2008) and dual Cre/Flp-responsive reporter and effector alleles (Dymecki et al., 2010; Ray et al., 2011; Brust et al., 2014; Niederkofler et al., 2016) to label Tac1-Pet1 boutons, identifying innervation targets, or to suppress Tac1-Pet1 neuron excitability, examining effects on the respiratory response to hypercapnia.

\section{Materials and Methods}

Transgenic mouse strains. Procedures were in accordance with Institutional Animal Care and Use Committee policies at Harvard Medical School and Dartmouth Giesel School of Medicine. Triple-transgenic animals were generated by breeding Tac1::IRES-cre (Harris et al., 2014) mice to either double-transgenic Pet1::Flpe (Jensen et al., 2008), RC:: FPDi (Ray et al., 2011) mice, Pet1::Flpe, RC::FrePe (Brust et al., 2014) mice, or Pet1::Flpe; RC::FPSiT (Niederkofler et al., 2016) mice. Doubleand single-transgenic sex-matched littermates served as controls.

Respiratory assays. Nonanesthetized adult Tac1::IRES-cre, Pet1::Flpe, $R C:: F P D i$ male and female mice $(n=18)$ and littermate controls $(n=18)$ were acclimated individually for $20 \mathrm{~min}$ in a whole-body plethysmograph chamber filled with room air. The animals were then exposed to $5 \% \mathrm{CO}_{2}$ for $15 \mathrm{~min}$ and then switched back to room air for $15 \mathrm{~min}$. Mice were removed for an intraperitoneal injection of $10 \mathrm{mg} / \mathrm{kg} \mathrm{CNO}$ (1 $\mathrm{mg} / \mathrm{ml}$ in saline) and immediately returned to the chamber with room air for $10 \mathrm{~min}$, after which gas was changed to $5 \% \mathrm{CO}_{2}$ for $15 \mathrm{~min}$. The last 5 min of each segment were used for data analyses. Rectal thermocouple temperature was read immediately before placement in the chamber, upon removal for injection, and upon final removal and no significant deviations in temperature were noted in these experiments. The average of these three temperatures was used in the tidal volume calculation below. Tracings with high background noise due to animal movement (observed directly during the assay) were noted at the time of assay and not included in final data analysis.

Respiratory airflow was recorded in a $140 \mathrm{ml}$ water-jacketed, temperature-controlled, glass chamber attached to a differential pressure transducer (Validyne Engineering) and reference chamber. Water temperature was $35.1^{\circ} \mathrm{C}$, resulting in a chamber temperature of $34^{\circ} \mathrm{C}$. An AEI Technologies pump pulled air through the chamber at $\sim 325 \mathrm{ml} / \mathrm{min}$. Volume calibrations were performed by repeated known-volume injections. Humidified gas flowing into the chamber was either room air or a $5 \% \mathrm{CO}_{2}$ mixture balanced with medical grade air. Pressure transducer readings were digitized at $1 \mathrm{kHz}$ (PowerLab; ADInstruments) and analyzed offline for peak amplitude, peak frequency, and average voltage (LabChart 6; ADInstruments). Tidal volume was determined by the following formula:

$$
\begin{aligned}
\{[(\mathrm{A} / \mathrm{B}) \times \mathrm{C}] \times[(\mathrm{D} & +273.15) \times(\mathrm{F}-\mathrm{H})]\} /\{[(\mathrm{D}+273.15) \\
& \times(\mathrm{F}-\mathrm{H})]-[(\mathrm{E}+273.15) \times(\mathrm{F}-\mathrm{G})]\}
\end{aligned}
$$

Where $A$ is the peak of breath signal (volts), $B$ is the peak of signal for injection volume, $C$ is the volume injection $(\mathrm{ml}), D$ is the body temperature $\left({ }^{\circ} \mathrm{C}\right), E$ is the chamber temperature $\left({ }^{\circ} \mathrm{C}\right), F$ is the barometric pressure $(\mathrm{mmHg}), G$ is the pressure of the water vapor of the mouse $=$ $1.142+(0.8017 \times D)-\left(0.012 \times D^{2}\right)+\left(0.0006468 \times D^{3}\right)$, and $H$ is the pressure of the water vapor of the chamber $=1.142+(0.8017 \times E)-$ $\left(0.012 \times E^{2}\right)+\left(0.0006468 \times E^{3}\right)$

Results were analyzed by two-way ANOVA with Holm-Sidak correction for multiple comparisons, with inspired gas and $\mathrm{CNO}$ administration as factors (GraphPad Prism). After significance was identified in two-way ANOVA, post hoc paired $t$ tests were performed. The extent to which ventilation, $\dot{V}_{\mathrm{E}}\left(\mathrm{ml} / \mathrm{g} / \mathrm{min}\right.$ ) response to $5 \% \mathrm{CO}_{2}$ (the percentage increase), was reduced upon $\mathrm{CNO} / \mathrm{Di}$-induced neuronal inhibition, referred to as the "difference value," was calculated as follows: $\left[\left(\dot{V}_{\mathrm{E}}^{5 \% \mathrm{CO} 2} / \dot{V}_{\mathrm{E}}^{\text {Room Air }}\right) \times 100 \%-100 \%\right]_{\text {pre-CNO }}-\left[\left(\dot{V}_{\mathrm{E}}^{5 \% \mathrm{CO} 2} / \dot{V}_{\mathrm{E}}^{\text {Room Air }}\right) \times\right.$ $100 \%-100 \%]_{\text {post-CNO. }}$

Temperature assay. Male Tac1-IRES::Cre, Pet1::Flpe, RC::FPDi and littermate control animals were implanted with IPTT-300 transponders (Bio-Medic Data Systems) subcutaneously at least $3 \mathrm{~d}$ before experiments. Temperatures were obtained via scanning with a Bio Medic Data Systems transponder reader without handling the animal. On the day of the experiment, animals were singly housed in cages with bedding and ad libitum access to food and water. Animals were weighed and placed into 
their cages and allowed a 30-60 min period of acclimatization. No anesthetic or analgesic reagents were used in these experiments. Baseline temperature was measured at 0,15 , and $30 \mathrm{~min}$. After the $30 \mathrm{~min}$ time point, an intraperitoneal injection of $10 \mathrm{mg} / \mathrm{kg}$ CNO $1 \mathrm{mg} / \mathrm{ml}$ dissolved in saline was performed. After injection, animals remained at room temperature for $30 \mathrm{~min}$ and body temperature recorded. Animals were then transferred to $4^{\circ} \mathrm{C}$ for $2 \mathrm{~h}$, with body temperatures measured every 10 min for the first hour and every $30 \mathrm{~min}$ for the second hour.

Tissue collection and sectioning. For fixed tissue, adult mice were anesthetized with Avertin (tribromoethanol) and immediately perfused intracardially with PBS followed by $4 \%$ paraformaldehyde (PFA) in PBS. Tissue was fixed overnight in $4 \% \mathrm{PFA}$ at $4^{\circ} \mathrm{C}$. Brains were then cryoprotected using 30\% sucrose in PBS and subsequently embedded in TissueFreezing Medium (Triangle Biomedical Sciences) and six sets of $20 \mu \mathrm{m}$ on-slide or $40 \mu \mathrm{m}$ free-floating serial coronal sections were collected in PBS. For fresh tissue, adult mice were anesthetized with Avertin and decapitated and brains were collected and subsequently embedded in Tissue-Tek Optimal Cutting Temperature Compound (Sakura Finetek). Embedded tissue was flash-frozen in 2-methylbutane on dry ice and 6 sets of $20 \mu \mathrm{m}$ on-slide coronal sections were collected.

Immunohistochemistry. For transgenic $R C::$ FrePe tissue, free-floating and on-slide sections were rinsed with $0.1 \%$ Triton X-100 PBS (PBS-T) and blocked in $5 \%$ normal donkey serum in PBS-T for $1 \mathrm{~h}$ at room temperature, followed by $24-48 \mathrm{~h}$ incubation at $4^{\circ} \mathrm{C}$ with primary antibody in PBS-T (chicken polyclonal anti-GFP, 1:5000, ab13970, Abcam and rabbit polyclonal anti-dsRed, 1:1000, catalog \#632496, Clontech). Sections were then washed with PBS-T three times and incubated with secondary antibody (IgG-Alexa Fluor 488-conjugated donkey antichicken secondary antibody, 1:500, Jackson ImmunoResearch Laboratories) and Cy3-conjugated donkey anti-rabbit secondary antibody (1:500; Jackson ImmunoResearch Laboratories). Cell nuclei were visualized with DAPI. For transgenic RC::FPSiT tissue, free-floating sections were prepared as above. Each set was stained with chicken polyclonal anti-GFP (1:2000, ab13970; Abcam) and rabbit polyclonal anti-tyrosine hydroxylase (TH) (1:5000, AB152; Millipore), rabbit polyclonal anti-NK1R (1: 2000, S8305; Sigma-Aldrich), or goat polyclonal anti-choline acetyltransferase (ChAT) (1:500, AB144P; Millipore). Primary antibody incubation was performed for $48-72 \mathrm{~h}$ at $4^{\circ} \mathrm{C}$ in PBS-T with $5 \%$ serum (NK1R, TH) or without (ChAT). Sections were then washed with PBS-T 3 times and incubated with secondary antibody at room temperature for $2 \mathrm{~h}$. Secondary antibody staining was performed with IgG-Alexa Fluor 488-conjugated donkey anti-chicken (1:500; Jackson ImmunoResearch Laboratories) and either Cy3-conjugated donkey anti-rabbit (1:500; Jackson ImmunoResearch Laboratories) or Cy3-conjugated donkey anti-goat 1:500; Jackson ImmunoResearch Laboratories). Cell nuclei were visualized with DAPI.

Phox $2 b$ and ChAT staining. For transgenic RC::FPSiT tissue, on-slide cryosections were rinsed 3 times with PBS for $10 \mathrm{~min}$ and submitted to steamer antigen-retrieval in $10 \mathrm{~mm}$ sodium citrate, $\mathrm{pH}$ 6.0, for $15 \mathrm{~min}$. After cooling down at room temperature (RT) for $20 \mathrm{~min}$, the sections were washed 5 times in PBS for $5 \mathrm{~min}$, permeabilized with $0.5 \%$ Triton $\mathrm{X}-100$ in PBS for $2 \mathrm{~h}$, and quenched in $0.1 \mathrm{~m}$ glycine for $30 \mathrm{~min}$ at RT. The sections were then rinsed 3 times with antibody buffer $(0.2 \%$ gelatin, 300 $\mathrm{mm} \mathrm{NaCl}, 0.3 \%$ Triton X-100 in PBS) for $5 \mathrm{~min}$, followed by incubation for $72 \mathrm{~h}$ at $4^{\circ} \mathrm{C}$ with the primary antibodies in the same buffer. Primary antibodies were as follows: chicken polyclonal anti-GFP (1:1000; GFP1010; Aves Labs), mouse monoclonal anti-Phox2b (1:100, B-11, sc376997; Santa Cruz Biotechnology), and goat polyclonal anti-ChAT (1: 100, AB144P; Millipore). Sections were then washed with antibody buffer 3 times for $5 \mathrm{~min}$ and incubated with secondary antibodies for $2 \mathrm{~h}$ at RT. Secondary antibodies were as follows: donkey anti-chicken IgGAlexa Fluor 488 (1:500; Jackson ImmunoResearch Laboratories), donkey anti-mouse IgG-Alexa Fluor 594 (1:500; Thermo Fisher Scientific), and donkey anti-goat IgG-Alexa Fluor 647 (1:500; Thermo Fisher Scientific). DAPI was used for nuclear counterstaining.

RNAscope in situ hybridization. Fresh-frozen brain tissue from adult Tac1::IRES-cre, Pet1::Flpe, RC::FrePe mice was collected as described above. Slides were postfixed with 4\% PFA, dehydrated in EtOH, and stained with the RNASCOPE Multiplex Fluorescent Assay Kit (Advanced Cell Diagnostics) as described previously (Wang et al., 2012) with $e G F P$ (catalog \#400281) and Tac1 (catalog \#410351-C3) probes. Cell nuclei were visualized with DAPI.

Image collection. Epifluorescence images were collected using an upright Zeiss Axioplan2 microscope equipped with an Axiocam 506 camera. SlideScanner epifluorescence images were collected on a VS120-SL microscope. SlideScanner images were brightness and contrast adjusted uniformly across the entire image in FIJI (http://fiji.sc/Fiji) for ease of visualization. Confocal $z$-stacks were collected on a Zeiss LSM 780 inverted microscope; for RC::FPSiT tissue, laser power and pinhole diameter were optimized for the GFP channel and kept constant for all images collected. Three triple-transgenic animals and double-transgenic control animals from independent litters, aged postnatal day 60 (P60), were used. Two equivalent anatomical regions were selected for each target area from each animal imaged. Efforts were made to select equivalent areas across samples using the anatomical landmarks described here. ChAT immunostaining was used to mark motor nuclei [nucleus ambiguus (NA), trigeminal nucleus $(5 \mathrm{~N})$, facial nucleus $(7 \mathrm{~N})$, spinal accessory nucleus $(11 \mathrm{~N})$, and hypoglossal nucleus $(12 \mathrm{~N})]$. In general, only one slice contained the $11 \mathrm{~N}$ per series, whereas the $5 \mathrm{~N}, 7 \mathrm{~N}$, and $12 \mathrm{~N}$ images were collected where the region of ChAT staining was the largest, marking the middle of their anterior-posterior extent. For the pre-Bötzinger complex (preBötC), the compact NA was used as an anatomical landmark because both are NK1R immunoreactive. For the parabrachial/pericoerulear region, images were taken immediately adjacent to the crescent-shaped locus ceruleus, marked by TH, where the dendrites of these neurons were visible. For both the caudal and rostral nucleus of the solitary tract (NTS), TH-positive cell bodies were used to mark the region; rostral NTS images were collected adjacent to the fourth ventricle, whereas caudal NTS images were collected adjacent to the area postrema and central canal, taking care to avoid the dorsal motor nucleus of the vagus. The $\mathrm{Cl}$ adrenergic nucleus was visualized with $\mathrm{TH}$ and was generally only present in one slice per series. Image stacks (.czi files) were imported into FIJI for background subtraction and thresholding (using a minimum fluorescence intensity of 15) to generate GFP mask images. Control images collected from each region had negligible fluorescence intensity signal above the selected threshold.

\section{Results}

Tac1-Pet1 intersectionally labeled neurons reside primarily in the raphe obscurus, raphe pallidus, and lateral paragigantocellularis and express Tac1 transcript

To ascertain whether Pet $1:$ Flpe-expressing raphe neurons have a history of Tacl expression by adulthood and to determine where these neurons are located, we used an intersectional genetic strategy that combined a Tac1::IRES-cre knock-in allele (Harris et al., 2014), a Pet1::Flpe BAC transgenic driver (Jensen et al., 2008), and the intersectional reporter allele RC::FrePe (Bang et al., 2012; Brust et al., 2014) (Fig. 1A) to mark double-positive cells with eGFP (referred to as the intersectional population, or in this case, the Tac1-Pet1 subtype) while simultaneously marking all other Pet1::Flpe-expressing raphe cells with mCherry (referred to as the subtractive Pet1 neuron population). The majority of eGFP+ neurons were found populating the full rostrocaudal extent of the raphe obscurus, as well as the immediately ventral raphe pallidus, albeit to a lesser extent (Fig. $1 G$ ). These findings are consistent with reported transcriptional profiling studies of Pet1 neurons isolated from these anatomical regions (Okaty et al., 2015), as well as immunohistochemical studies localizing substance $\mathrm{P}$ (Ptak et al., 2009). Also in the medulla, a subset of intersectionally labeled eGFP + cells were found in the caudal portion of the raphe magnus and the lateral paragigantocellularis (Fig. $1 E, F$ ); in contrast, in the midbrain/pons region, only a few eGFP + cells were detected in the dorsal raphe (Fig. 1C) and none within the median raphe (Fig. 1D). In addition to adult tissue, we examined P8 and P15 brain tissue from triple-transgenic Tac1::IRES-cre, Pet $1:: F l p e, R C:: F r e P e$ animals and identified eGFP + cells in the 

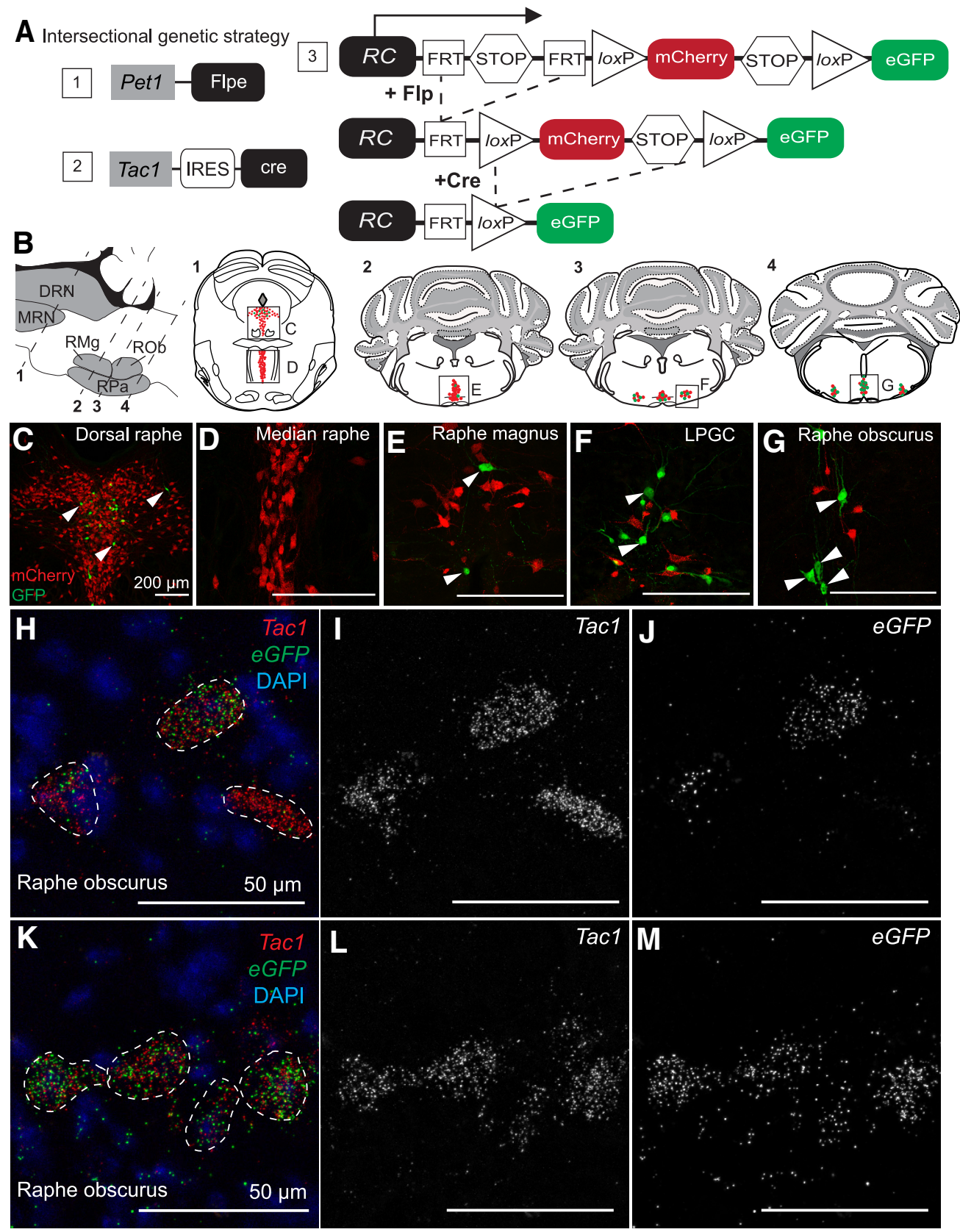

Figure 1. Tac1-Pet1 neurons are located primarily in caudal medullary raphe nuclei and express Tac1 transcript. $\boldsymbol{A}$, Intersectional genetic strategy: a combination of expressed transgenes 1 and 2 result in dual Flpe- and Cre-mediated recombination of intersectional allele, RC::FrePe (knock-in transgene 3) in cells expressing both Tac 1 and Pet1. B, Sagittal and coronal schematics of mouse brainstem slices highlighting anatomical locations of 5 -HT raphe nuclei. Intersectionally labeled eGFP-positive cells were found in the dorsal raphe $(\boldsymbol{C})$, caudal raphe magnus $(\boldsymbol{E})$, lateral paragigantocellularis $(\boldsymbol{F})$, and raphe obscurus $(\boldsymbol{G})$, but not in the median raphe, (D); mCherry marks other (non-Tac1-expressing) Pet1 neurons. Scale bars, $200 \mu \mathrm{m}$. $\boldsymbol{H}-\boldsymbol{M}, T a c 1$ transcript (red) was localized within all eGFP transcript-positive (green) cells examined in the raphe obscurus in Tac1::IRES-cre, Pet1::Flpe, RC::FrePe tissue. Tac 1 transcript $(\boldsymbol{I}, \boldsymbol{L})$, and eGFP transcript $(\boldsymbol{J}, \boldsymbol{M})$ shown separately in grayscale. Scale bars, $50 \mu \mathrm{m}$.

dorsal raphe and the raphe obscurus at P8 and P15 (data not shown), indicating that Tac1::IRES-cre expression in Pet1::Flpepositive cells is initiated by $\mathrm{P} 8$.

Dual Flpe- and Cre-mediated recombination of the RC::FrePe reporter allele indicates a history and/or possible time-of-harvest expression of the recombinases. To assess adult, time-of-harvest Tacl expression in these Pet1 neurons, we used RNAscope in situ hybridization to detect Tacl and eGFP mRNA in tripletransgenic adult Tac1::IRES-cre, Pet1::Flpe, RC::FrePe tissue. In all $e G F P+$ cells (Tac1-Pet1 lineage cells) examined, in this case in the raphe obscurus, $T a c 1$ transcripts were detected, suggesting active adult expression of Tachykinin 1 and substance P. Some nearby neurons expressed Tacl, but little to no eGFP (Fig. $1 H-M$ ), indicating expression of Tacl in raphe neurons not captured by our Pet1::Flpe driver; these Tac1 + neurons could represent non5-HT neurons expressing Tac1 or Pet1 neurons missed by Pet1:: Flpe, which are are known to exist (Barrett et al., 2016).

Acute silencing of Tac1-Pet1 neurons blunts the $5 \% \mathrm{CO}_{2}$ respiratory chemoreflex

The respiratory chemoreflex underlies the increase in ventilation required to clear elevated tissue/blood $\mathrm{CO}_{2}$ levels to maintain 


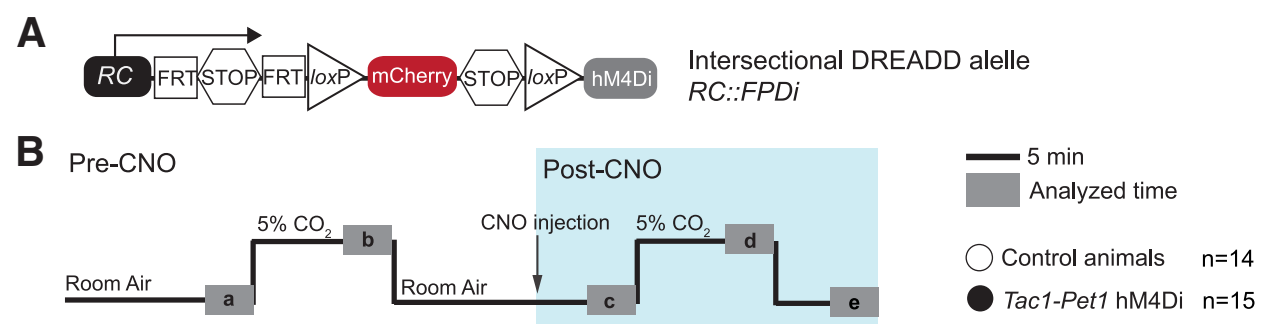

C

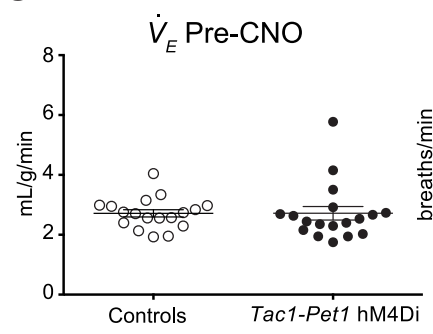

D

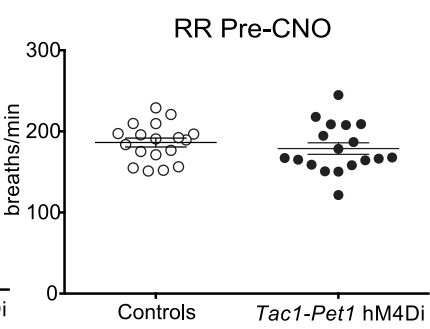

$\mathrm{E}$

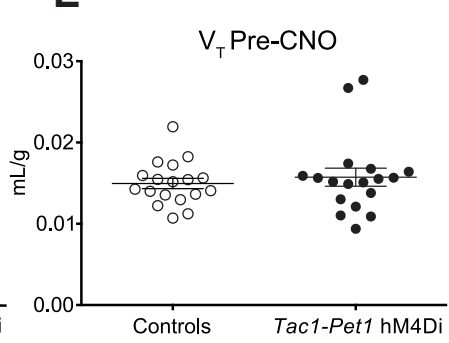

$\mathbf{F}$

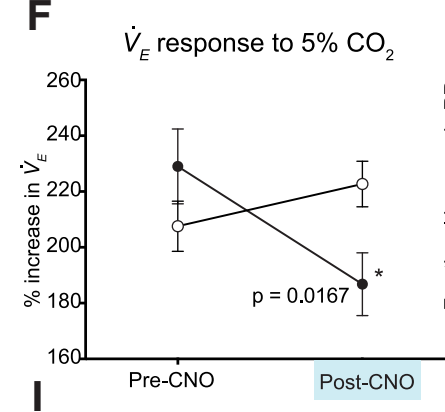

G
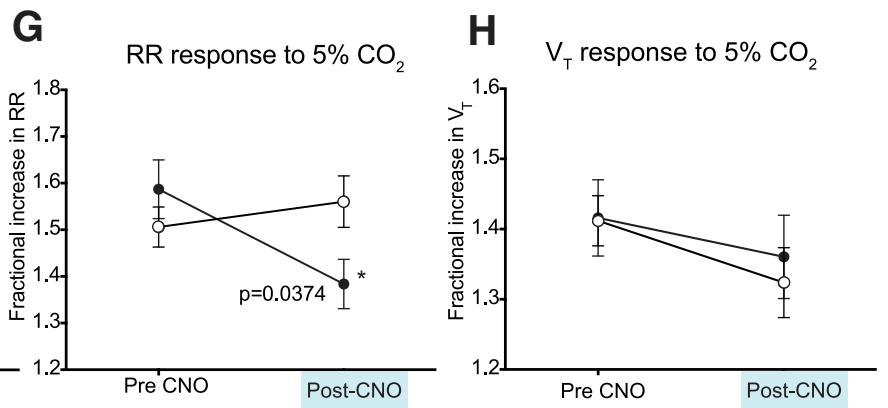

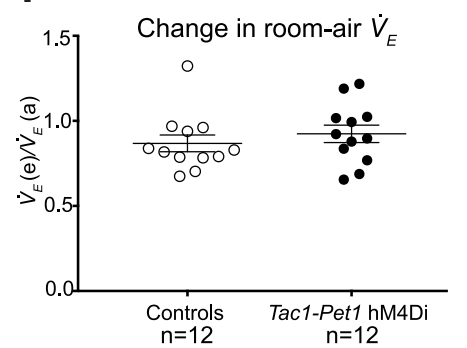

Figure 2. Acute silencing of Tac1-Pet1 neurons results in a decrease in ventilation at $5 \% \mathrm{CO}_{2} . \mathbf{A}$, Intersectional DREADD allele. $\boldsymbol{B}$, Experimental design. After an initial period of acclimation, experimental adult animals and littermate controls are exposed to $5 \% \mathrm{CO}_{2}$ and then returned to room air, briefly removed from the chamber for intraperitoneal injection of $10 \mathrm{mg} / \mathrm{kg} \mathrm{CNO}$, and placed back in room air. Animals were then exposed to $5 \% \mathrm{CO}_{2}$ a second time, followed by another period of room air breathing in 12 animals from each group. Gray boxes ( $\left.\boldsymbol{a}-\boldsymbol{d}\right)$ indicate time intervals used for data analysis. $\mathbf{C}-\boldsymbol{E}$, Baseline measurements of $\dot{V}_{\mathrm{E}}$, respiratory rate $(\mathrm{RR})$, and tidal volume $\left(V_{\mathrm{T}}\right)$ in control $(n=14)$ and Tac1::IRES-cre, Pet1:::Flpe, RC::.FPDi ("Tac1-Pet1 hM4Di") animals $(n=15)$ before CNO injection were not significantly different. Each circle represents one animal, error bars represent SEM. $F$, Average percentage increase in $\dot{V}_{\mathrm{E}}$ before $\mathrm{CNO}$ injection (pre-CNO, $\dot{V}_{\mathrm{E}}$ b $/ \dot{V}_{\mathrm{E}}$ a $\times 100 \%$ ) and minutes after CNO injection (post-CNO, $\dot{V}_{\mathrm{E}}^{\mathrm{d}} / \dot{V}_{\mathrm{E}}^{\mathrm{c}} \times 100 \%$ ) in control and Tac1-Pet1 hM4Di animals. Error bars indicate SEM. G, $\boldsymbol{H}$, Average fractional increase in respiratory rate (RR) or tidal volume $\left(V_{\mathrm{T}}\right)$ to $5 \% \mathrm{CO}_{2}$ before and after $\mathrm{CNO}$ injection. $I$, Change in room air ventilation after CNO administration compared with baseline $\left(\dot{V}_{\mathrm{E}}^{\mathrm{e}} / \dot{V}_{\mathrm{E}}{ }^{\mathrm{a}}\right)$ in control $(n=12)$ and Tac1-Pet1 hM4Di animals $(n=12)$. See Results for numerical data and statistical tests.

blood pH homeostasis (Dean and Nattie, 2010; Guyenet et al., 2010; Nattie and Li, 2012). 5-HT neuron function is required to mount a robust chemoreflex, both in models in which 5-HT neurons fail to develop (Hodges et al., 2008) and in models of acute suppression of Pet1::Flpe- or Slc6a4::cre-expressing neurons (Ray et al., 2011). Selective suppression of just the Egr2-Pet1 5-HT neuron subtype comparably blunts the chemoreflex as well, whereas most other Pet1 neuron subtypes do not (Brust et al., 2014); Tac1-Pet1 neurons were not analyzed previously due to lack of a suitable driver. Now with the availability of the Tac1:: IRES-cre driver (Harris et al., 2014) to couple with Pet1::Flpe, we applied our dual-recombinase intersectional $R C:: F P D i$ allele (Fig. 2A) to selectively and acutely perturb Tac1-Pet1 neurons in vivo in the context of hypercapnia. $R C:: F P D i$ is identical in design to the RC::FrePe allele (Bang et al., 2012; Brust et al., 2014) used above except that, in $R C:: F P D i$, the synthetic inhibitory receptor hM4Di (Armbruster et al., 2007) is expressed in the Cre/Flpe dual-targeted subtype, whereas mCherry marks remainder of the Pet1 neurons (the subtractive population; Ray et al., 2011; Brust et al., 2014). Upon binding of its exogenous ligand CNO, hM4Di triggers cellautonomous hyperpolarization and reduced neuronal excitability (Armbruster et al., 2007; Ray et al., 2011; Brust et al., 2014). Di has been used previously to suppress excitability of Pet1 neurons in primary neuronal cultures, transgenic brainstem slices, and transgenic mice (Ray et al., 2011; Brust et al., 2014; Teissier et al., 2015; Brust and Dymecki, unpublished data) and has been shown to inhibit synaptic transmission 
(Stachniak et al., 2014). Here, we targeted Di expression to allow CNO-triggered inhibition selectively in Tac1-Pet1 neurons.

We used whole-body plethysmography to assay the ventilatory response to inspired $5 \% \mathrm{CO}_{2}$ before and during acute $\mathrm{CNO} /$ Di-triggered perturbation of Tac1-Pet1 neurons (Fig. 2B) as described previously (Ray et al., 2011; Brust et al., 2014). Mice were assayed in quiet wakefulness because vigilance state has been shown to affect the respiratory chemoreflex in some models $(\mathrm{Na}-$ kamura et al., 2007). Body temperature was also monitored and found to be stable during these measurements; the plethysmograph chamber was held continuously at $34^{\circ} \mathrm{C}$. To test the effects of Tac-Pet 1 neuron activity perturbation on ventilation, animals were exposed to a series of $\mathrm{CO}_{2}$ challenges and their ventilation measured as diagrammed in Figure $2 B$. Before $\mathrm{CNO}$ administration (at time point "a"), no significant differences were found in the baseline respiratory rate, tidal volume, ventilation $\left(\dot{V}_{\mathrm{E}}\right)$, or oxygen consumption $\left(\dot{V}_{\mathrm{O} 2}\right)$ of the Tac1-Pet1 hM4Di-expressing mice compared with littermate controls, indicating relative neutrality of the hM4Di receptor on respiration in the absence of $\mathrm{CNO}$ (Fig. 2C-E). Before $\mathrm{CNO}$ injection, during the first $\mathrm{CO}_{2}$ challenge, the percentage increase in $\dot{V}_{\mathrm{E}}$ upon $5 \% \mathrm{CO}_{2}$ exposure (from time point "a" to "b") was not significantly different between Tac1-Pet1 hM4Di-expressing mice and control littermates (229\% vs $207.6 \%$, unpaired $t$ test, $p=0.20$ ) (Fig. $2 F$, pre-CNO). However, upon CNO administration, a significant blunting of the ventilatory response to $5 \% \mathrm{CO}_{2}$ (from time point " $c$ " to " $\mathrm{d}$ ") was observed in only the Tac1-Pet1 hM4Di-expressing mice (229\% vs $186.8 \%$, paired $t$ test, $p=0.0167, t=2.717$, df $=14$ ), whereas the ventilatory response of control littermates showed no significant difference from pre-CNO measurements $(207.6 \%$ vs $222.7 \%$, paired $t$ test, $p=0.27$ ) (Fig. $2 F$, post-CNO).

We then examined increases in respiratory rate and tidal volume separately to ascertain whether perturbation of Tac1-Pet1 neuron activity affected these two components of ventilation differentially. The fractional increase in respiratory rate in response to $5 \% \mathrm{CO}_{2}$ was significantly blunted in Tac1-Pet1 hM4Diexpressing mice upon $\mathrm{CNO}$ exposure compared with pre-CNO levels ( 1.38 vs 1.56 , paired $t$ test, $p=0.0374, t=2.269$, df $=16$ ), whereas the fractional increase in tidal volume in response to $5 \%$ $\mathrm{CO}_{2}$ was not significantly different upon Tac1-Pet1 neuron perturbation (Fig. 2G,H). In control littermates, the fractional increases in respiratory rate and tidal volume in response to $5 \%$ $\mathrm{CO}_{2}$ were not significantly altered upon $\mathrm{CNO}$ administration (Fig. 2G,H).

Although these experiments were not designed to best measure the effect of Tac1-Pet1 neuron suppression on room air ventilation (rather, the time course was optimized to assess effects on the chemoreflex), we nonetheless also examined the overall effect of CNO/hM4Di-mediated perturbation on room air ventilation in both control and Tac1-Pet1 hM4Di-expressing animals over the course of the experiment. Room air ventilation at time point " $e$ " and time point "a" was calculated and the e/a ratio determined for each animal, importantly allowing each animal to serve as its own control (Fig. 2I). Each group, control and hM4Diexpressing, showed a similar average ratio ( 0.87 vs 0.92 , unpaired $t$ test, $p=0.4375)$, thus revealing no discernable effects on room air ventilation.

Intact body temperature homeostasis in the face of Tac1-Pet1 neuron perturbation

Whereas acute silencing of all serotonergic neurons en masse results in rapid and significant hypothermia (Ray et al., 2011), this was not observed upon perturbation of the Tac1-Pet1 neuron subset. Specifically, after intraperitoneal injection of $10 \mathrm{mg} / \mathrm{kg}$ CNO followed by exposure to ambient $4^{\circ} \mathrm{C}$, Tac1-Pet 1 hM4Diexpressing male animals $(n=8)$, on average, dropped a maximum of $2.36 \pm 0.32^{\circ} \mathrm{C}$ body temperature, an effect similar to that observed for control male littermates $(n=8)$, which showed a maximum drop of $2.73 \pm 0.31^{\circ} \mathrm{C}$ (unpaired $t$ test, $p=0.42$ ).

\section{Tac1-Pet1 neurons project to motor nuclei and the preBötC respiratory rhythm generator}

Finding that Tac1-Pet1 neuron activity perturbation blunts the ventilatory response to hypercapnia (Fig. 2) and that Tac1-Pet1 neurons are located primarily within the raphe obscurus, a region known to project to motor nuclei within the brainstem (Loewy, 1981; Thor and Helke, 1987; Ellenberger et al., 1992; Depuy et al., 2011) (Fig. 1), we sought to identify brainstem and spinal cord regions receiving projections from Tac1-Pet1 neurons. We used the intersectional reporter allele $R C:: F P S i T$, which expresses a synaptophysin-GFP fusion in the intersectional neuron population (Niederkofler et al., 2016), to resolve Tac1-Pet1 axonal boutons within the brainstem and cervical spinal cord. Synaptophysin is a synaptic vesicle membrane glycoprotein that has been widely used as a marker of synaptic-vesicle-containing boutons (Nakata et al., 1998; Pennuto et al., 2002; Li et al., 2010). Upon dual recombination of the $R C:: F P S i T$ reporter allele, synaptophysin-GFP is expressed and trafficked cell autonomously to axonal boutons in intersectionally labeled neurons. This approach allows illumination of terminal and en passant axonal boutons upon fluorescent staining for GFP. In previous work examining the projection patterns of Pet1 raphe neurons, dorsal raphe Pet 1 neurons have been found to project mainly to forebrain areas, whereas medullary Pet1 raphe neurons projected within the brainstem and spinal cord (Bang et al., 2012). Although we cannot rule out that dorsal raphe Tac1-Pet1 neurons may contribute to brainstem projections, we presume that the majority of the innervation presented here originates from medullary Tac1-Pet1 neurons.

Fluorescent staining of synaptophysin-GFP labeled Tac1-Pet1 boutons revealed a pattern, which can be seen in low-magnification images (Fig. 3), of Tac1-Pet1 projections primarily within motor nuclei, some of which have roles in respiration and airway patency, including the $5 \mathrm{~N}, 7 \mathrm{~N}, 11 \mathrm{~N}, 12 \mathrm{~N}$, and NA. Within the cervical spinal cord, boutons were localized among the ventral motor neurons (VMNs), but not sensory neurons in the dorsal horn. A subset of these cervical spinal VMNs gives rise to the phrenic nerve, innervating the diaphragm, the main muscle of respiration. In contrast, the laterodorsal tegmental nucleus, which sends cholinergic projections to subcortical and cortical structures such as the thalamus and ventral tegmental area, was devoid of synaptophysin-GFP signal (Fig. $3 A$, inset), as was the dorsal motor nucleus of the vagus nerve $(10 \mathrm{~N})$ (Fig. $3 C$ ), which supplies parasympathetic motor innervation to various cells within the gastrointestinal tract.

After identifying this gross pattern of Tac1-Pet1 projections at low magnification (Fig. 3), we pursued a higher-resolution analysis using confocal microscopy to visualize terminals in motor regions where Tac1-Pet1 boutons were localized, as well as to explore chemosensory regions of the brainstem. Because nearby Egr2-Pet1 neurons were not found to send major projections to motor centers known to receive serotonergic innervation, but rather project heavily to chemosensory integration regions, including the retrotrapezoid nucleus (Brust et al., 2014), a known chemosensory site (Mulkey et al., 2015), our findings point to 

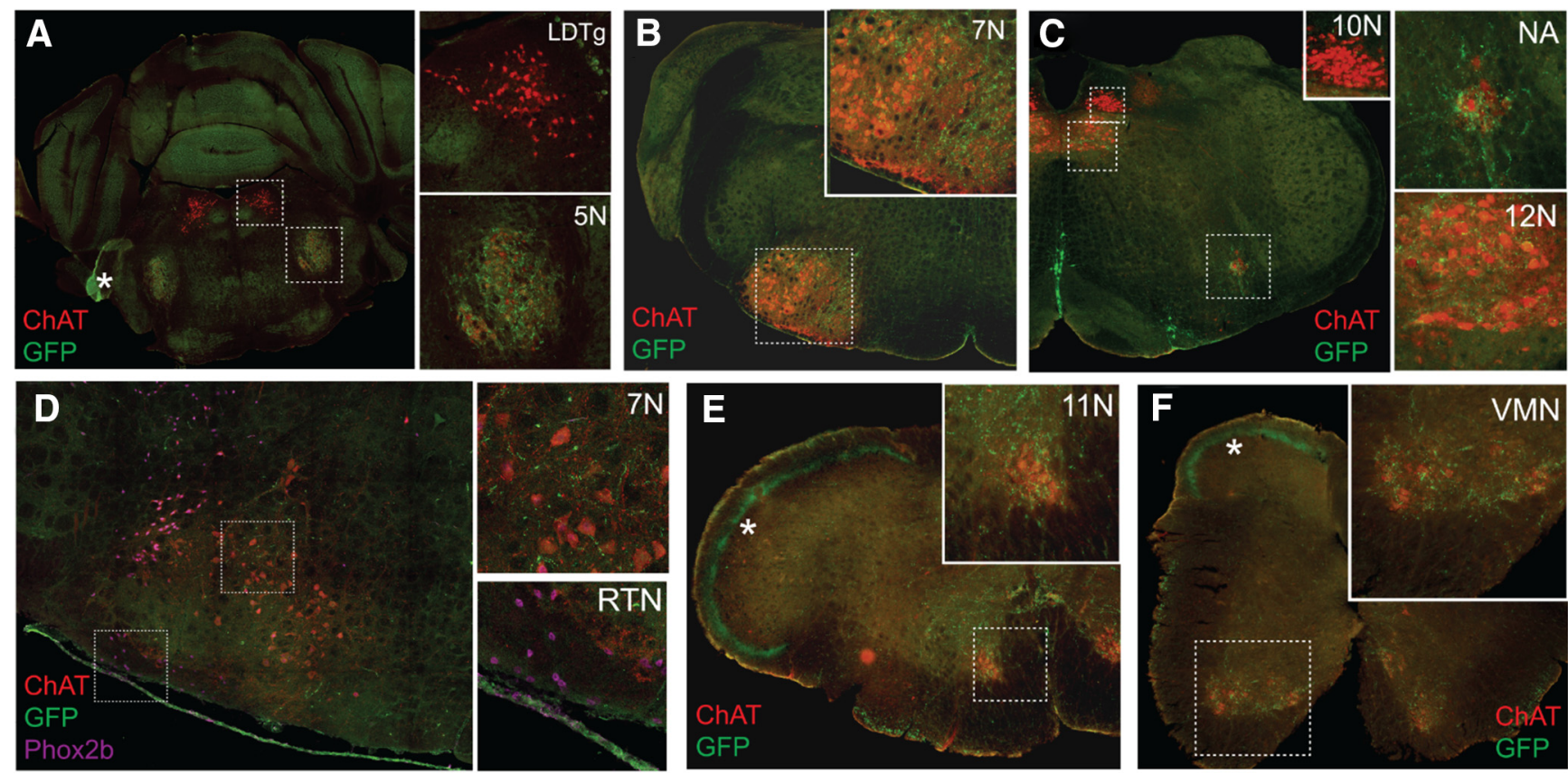

Figure 3. Tac1-Pet1 terminals primarily target respiratory and airway motor nuclei. Coronal brainstem sections from Tac 1::IRES-cre, Pet1::Flpe, RC::FPSiT animals were stained for synaptophysinGFP (green) to visualize terminals, ChAT (red) to identify motor neurons, and Phox2b (magenta) to mark retrotrapezoid nucleus neurons. A, $5 \mathrm{~N}$ receives Tac1-Pet1 projections, whereas the laterodorsal tegmental nucleus (LDTg) does not. B, 7N contains Tac 1-Pet 1 projections. C, $10 \mathrm{~N}$ does not receive projections, whereas $12 \mathrm{~N}$ and NA contain synaptophysin-GFP staining. $\boldsymbol{D}$, Retrotrapezoid nucleus (RTN) does not receive projections and lies ventral to the 7N, which is positive for Tac1-Pet1 projections. $\boldsymbol{E}, \boldsymbol{F}, 11 \mathrm{~N}(\boldsymbol{E})$ and VMNs $(\boldsymbol{F})$ in the cervical spinal cord both receive projections from Tac1-Pet1 neurons. In addition to marking axonal boutons, there is sufficient synaptophysin-GFP expression to mark Tac1-Pet1 cell bodies, as seen in $\boldsymbol{C}$. Marked by asterisks, prominent autofluorescence highlights the entering vestibulocochlear nerve tract in $\boldsymbol{A}$ and the dorsolateral spinocerebellar tracts in $\boldsymbol{E}$ and $\boldsymbol{F}$.

Tac1-Pet1 neurons as a source of serotonergic projections to motor nuclei.

We examined each of the motor nuclei identified in Figure 3 as receiving Tac1-Pet1 innervation, as well as chemosensory regions important in respiratory modulation. Each area was queried in three animals from independent litters; two equivalent anatomical regions were selected from each animal for imaging (see Materials and Methods). Representative images are shown to demonstrate relative density of synaptophysin-GFP-labeled terminals, alongside schematic coronal brainstem sections highlighting the area of interest (Fig. 4). GFP-mask images, thresholded and processed uniformly across all areas of GFP fluorescence data as described in the Materials and Methods section, are shown for ease of terminal visualization (Fig. $\left.4 C^{\prime \prime}-G^{\prime \prime}\right)$. TaclPet1 terminals were found in the trigeminal motor nucleus (Fig. $4 C$ ), where serotonergic input may contribute to the modulation of rhythmic oral activity (Hsiao et al., 2002), as well as the facial motor nucleus (Fig. 4D), which innervates skeletal muscles of the face and has been implicated in respiration in rodents (Zhang et al., 2004). Tac1-Pet1 terminal density was also found in the NA (Fig. 4E), which contains branchial motor neurons contributing to the vagus glossopharyngeal nerves, as well as the hypoglossal nucleus (Fig. $4 F$ ), which innervates the genioglossus, the main muscle of the tongue. The hypoglossal nucleus is critical for establishing airway patency, is activated during inspiration, and its output has been shown to be modulated by raphe obscurus neurons (Peever et al., 2001; Saboisky et al., 2006). Finally, we observed Tac1-Pet1 innervation in the spinal accessory nucleus (Fig. 4G), which innervates several of the accessory muscles of respiration. Because many of these regions innervate muscles involved in actions distinct from respiration, this suggests a possible broader role for Tac1-Pet1 neurons in motor regulation.
Given the role of Tac1-Pet1 neurons in the respiratory chemoreflex, we hypothesized that these neurons may also innervate brainstem regions involved in chemosensation and rhythm generation, perhaps at a lower terminal density than was seen in respiratory motor nuclei within the brainstem and spinal cord because these areas have been shown previously to receive dense innervation from the Egr2-Pet1 neuron subtype (Brust et al., 2014). We examined Tac1-Pet1 projections in several chemosensory regions of the brainstem using TH to identify cell bodies as anatomical landmarks for the parabrachial/pericoerulear region, the $\mathrm{C} 1$ adrenergic nucleus, and nucleus of the solitary tract and NK1R staining to mark the preBötC. Representative images are shown to illustrate qualitative innervation density to these regions (Fig. 5). The parabrachial/pericerulear region, imaged immediately lateral to the crescent-shaped locus ceruleus and containing dendrites from these neurons, was devoid of Tac1Pet1 innervation (Fig. 5 C), whereas this region is densely innervated by the Egr2-Pet1 subtype (Brust et al., 2014). The rostral and caudal NTS, responsible for the integration of respiratory stimuli from within the brainstem and from the periphery to coordinate cardiorespiratory control, and likely also chemosensitive themselves, received projections from Tac1-Pet1 neurons (Fig. $5 D, G$ ). The $\mathrm{C} 1$ adrenergic nucleus, which serves to modulate the sympathetic nervous system response to hypercapnia, also receives Tacl-Pet1 innervation (Fig. 5F). The preBötC, the primary respiratory rhythm generator, marked by NK1R staining, receives innervation from Tac1-Pet1 neurons as well (Fig. 5E). Overall, these chemosensory and rhythm generation centers received qualitatively lower terminal density from Tac1-Pet1 neurons than did the motor regions examined above, as can be seen in the thresholded GFP-mask images (Fig. 5 $C^{\prime \prime}-G^{\prime \prime}$ ). Notably, the chemosensory retrotrapezoid nucleus localized via Phox $2 \mathrm{~b}$-expressing cells 

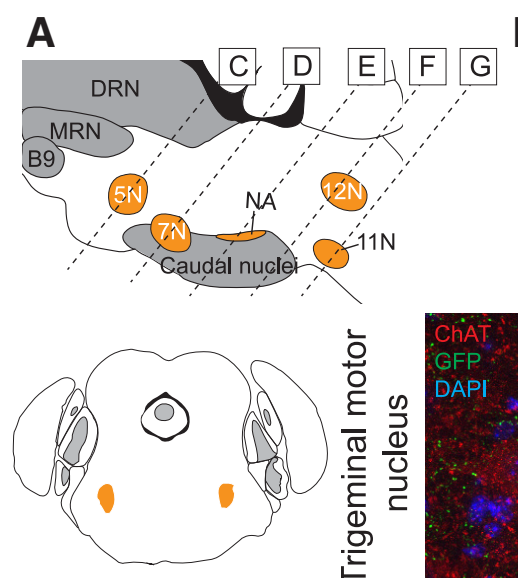

B
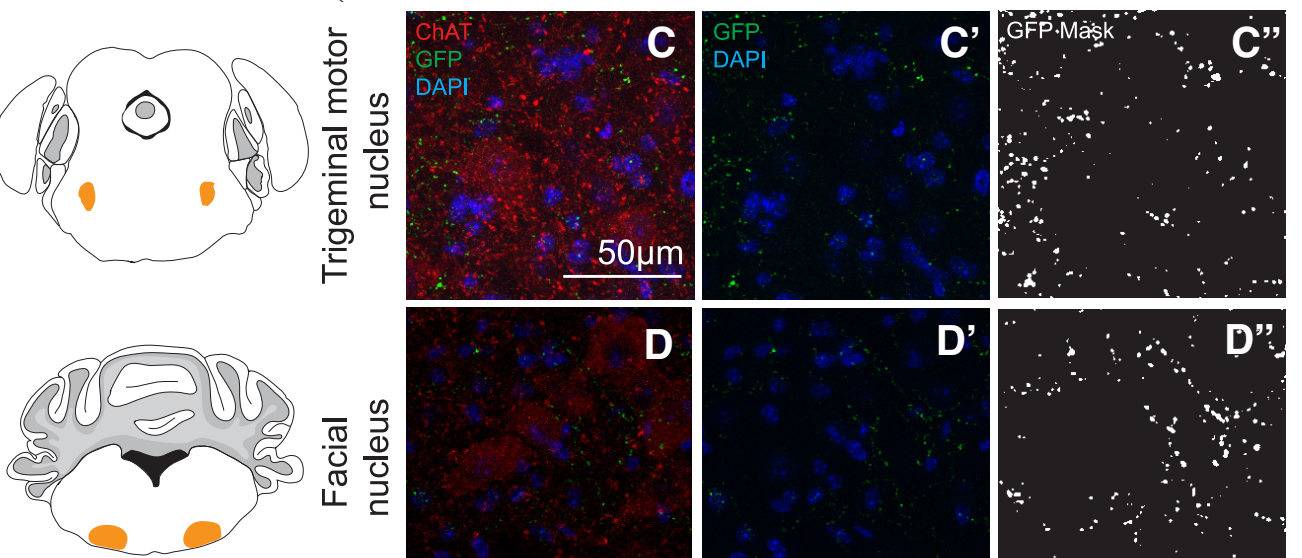

Intersectional synaptophysin-GFP allele, RC::FPSiT
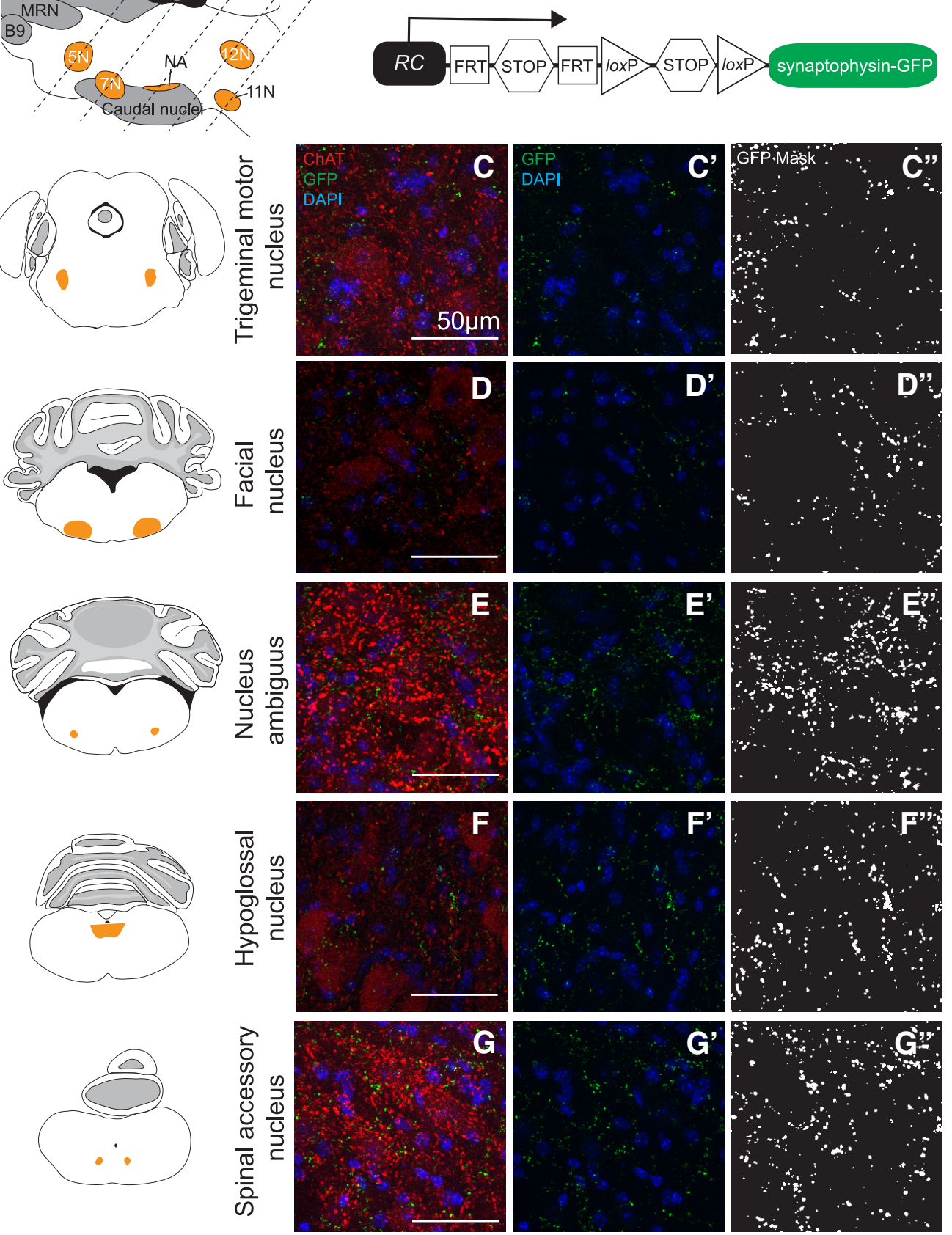

Figure 4. Tac1-Pet1 neurons project densely to specific motor nuclei within the brainstem. $\boldsymbol{A}$, Sagittal mouse brain schematic illustrating areas queried. $\boldsymbol{B}$, Intersectional $R C:: F P S i T$ allele. Representative images are shown with corresponding adjacent coronal brainstem schematic for the trigeminal motor nucleus $(\boldsymbol{C})$, facial nucleus $(\boldsymbol{D})$, NA $(\boldsymbol{E})$, hypoglossal nucleus $(\boldsymbol{F})$, and spinal accessory nucleus (G). Immunostained Tac 1::IRES-cre, Pet1::Flpe, RC::FPSiT tissue ( $n=3$ animals, $2-4$ confocal stacks per animal) are shown as maximum intensity projections of imaged confocal stacks, with synaptophysin-GFP puncta (green) from intersectional Tac1-Pet1 cells colocalized with ChAT immunostaining (red) and DAPI (blue) (C-G) and synaptophysin-GFP puncta with DAPI only $\left(\mathbf{C}^{\prime}-\mathbf{G}^{\prime}\right)$. In $\left(\mathbf{C}^{\prime \prime}-\mathbf{G}^{\prime \prime}\right)$, thresholded GFP mask images (see Materials and Methods) are shown for ease of visualization, highlighting the relative terminal density in regions throughout the brainstem. Scale bars, $50 \mu \mathrm{m}$.

ventral to the facial nucleus (Stornetta et al., 2006; Guyenet et al., 2010) and the associated lateral parafacial region involved in expiratory oscillation (Huckstepp et al., 2015) both contained little to no Tac1-Pet1 boutons (Fig. 3D).

\section{Discussion}

Diversity among serotonergic neurons is proving to be substantial (Jensen et al., 2008; Kim et al., 2009; Deneris, 2011; Hale and
Lowry, 2011; Gaspar and Lillesaar, 2012; Andrade and HajDahmane, 2013; Brust et al., 2014; Spaethling et al., 2014; Fernandez et al., 2016; Okaty et al., 2015; Niederkofler et al., 2016). Progress toward making sense of this heterogeneity has been aided by intersectional genetic tools that permit mechanistic probing of subsets of Pet1 raphe neurons (Jensen et al., 2008; Kim et al., 2009; Dymecki et al., 2010; Ray et al., 2011; Brust et al., 2014; Niederkofler et al., 2016); these tools are applied here to 
A

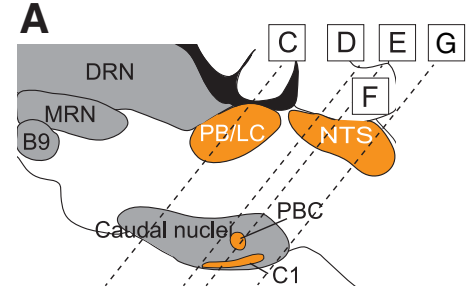

B

Intersectional synaptophysin-GFP allele, RC::FPSiT

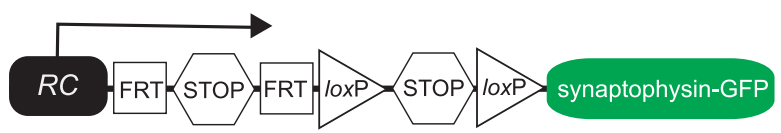

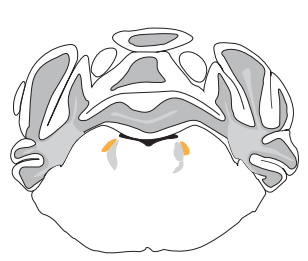
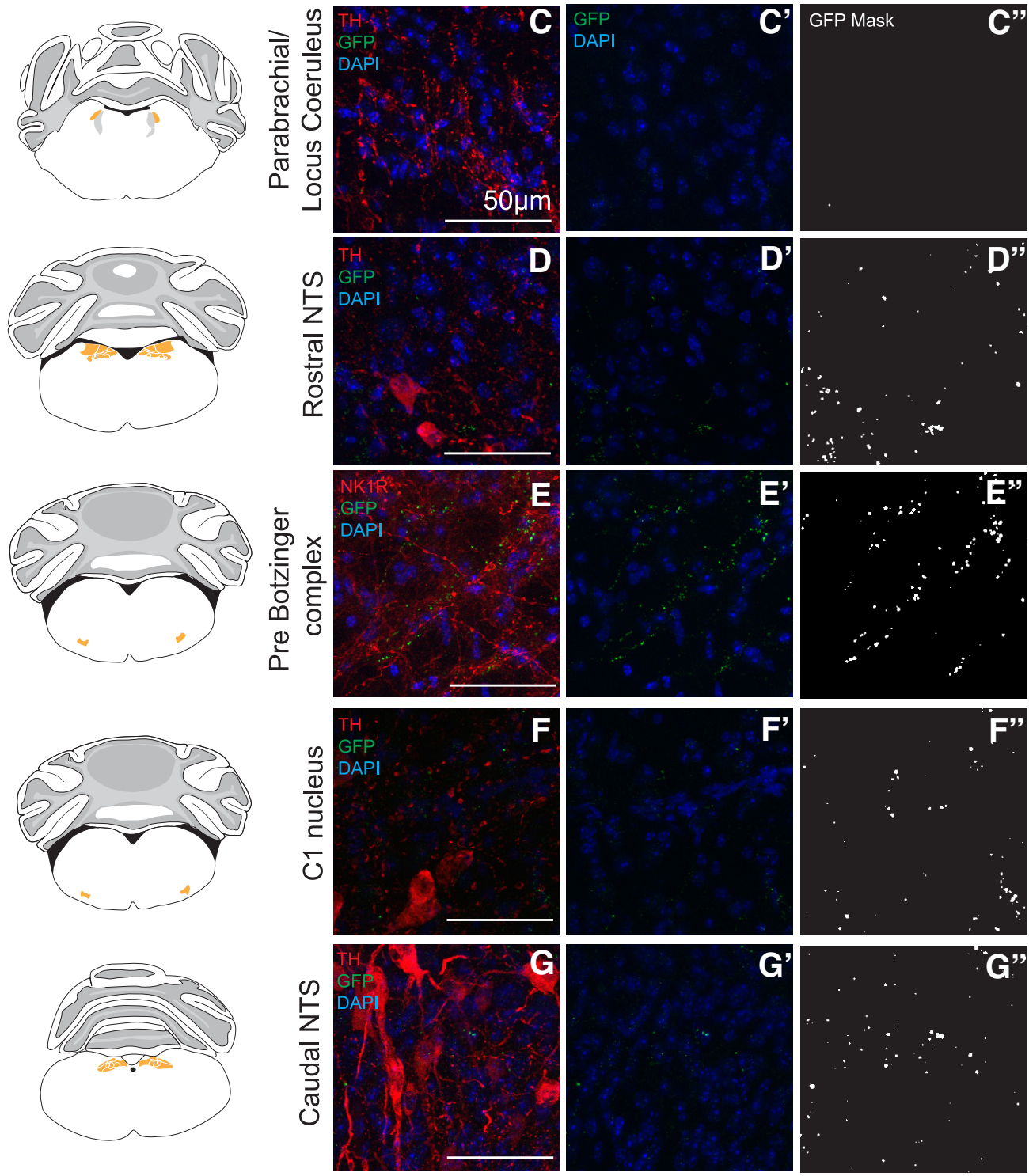

Figure 5. Tac1-Pet1 neurons project sparsely to chemosensory and rhythm generation regions within the brainstem. $\boldsymbol{A}$, Sagittal mouse brain diagram illustrating areas queried. $\boldsymbol{B}$, Intersectional $R C$ ::FPSiT allele. Representative images are shown with corresponding adjacent coronal brainstem schematic for the parabrachial/locus ceruleus region $(\boldsymbol{C})$, the rostral nucleus of the solitary tract (D), the preBötC $(\boldsymbol{E})$, the $C 1$ nucleus $(\boldsymbol{F})$, and the caudal NTS (G). Immunostained Tac1::IRES-cre, Pet1::Flpe, RC::FPSiT tissue $(n=3$ animals, $2-4$ confocal stacks per animal) are shown as maximum intensity projections of imaged confocal stacks, showing synaptophysin-GFP (green) labeled terminals colocalized with TH or NK1R immunostaining (red) and DAPI (blue) in (C-G). DAPI and GFP terminals alone are shown in $\left(\boldsymbol{C}^{\prime}-\boldsymbol{G}^{\prime}\right)$, and thresholded GFP mask images (see Materials and Methods) in $\left(\boldsymbol{C}^{\prime \prime}-\boldsymbol{G}^{\prime \prime}\right)$. Scale bars, $50 \mu \mathrm{m}$.

explore a newly accessible subtype of raphe neuron, the Tac1-Pet1 subtype. We show that CNO/hM4Di manipulation of Tac1-Pet1 neurons in vivo blunts the respiratory chemoreflex, suggesting a stimulatory effect of these neurons on breathing. Axonal boutons from Tac1-Pet1 neurons appear enriched in brainstem and spinal cord motor nuclei, many of which are necessary for normal respiration and airway patency. Tac1-Pet1 neurons are thus likely to exert a substantial portion of their effect through modulation of motor output (Fig. 6). These findings complement our previ- ously reported work on medullary raphe Egr2-Pet1 neurons. The latter, too, are required for a robust respiratory chemoreflex but, unlike Tac1-Pet1 neurons, Egr2-Pet1 neurons do not project to respiratory motor centers; rather, they innervate chemosensory centers preferentially and are themselves directly chemoreceptive to hypercapnic acidosis (Brust et al., 2014). In contrast, Tac1-Pet1 neurons likely do not function as direct chemoreceptors given the electrophysiological response properties observed for Pet1 neurons in the raphe obscurus region demarcated by Tac1 expression 

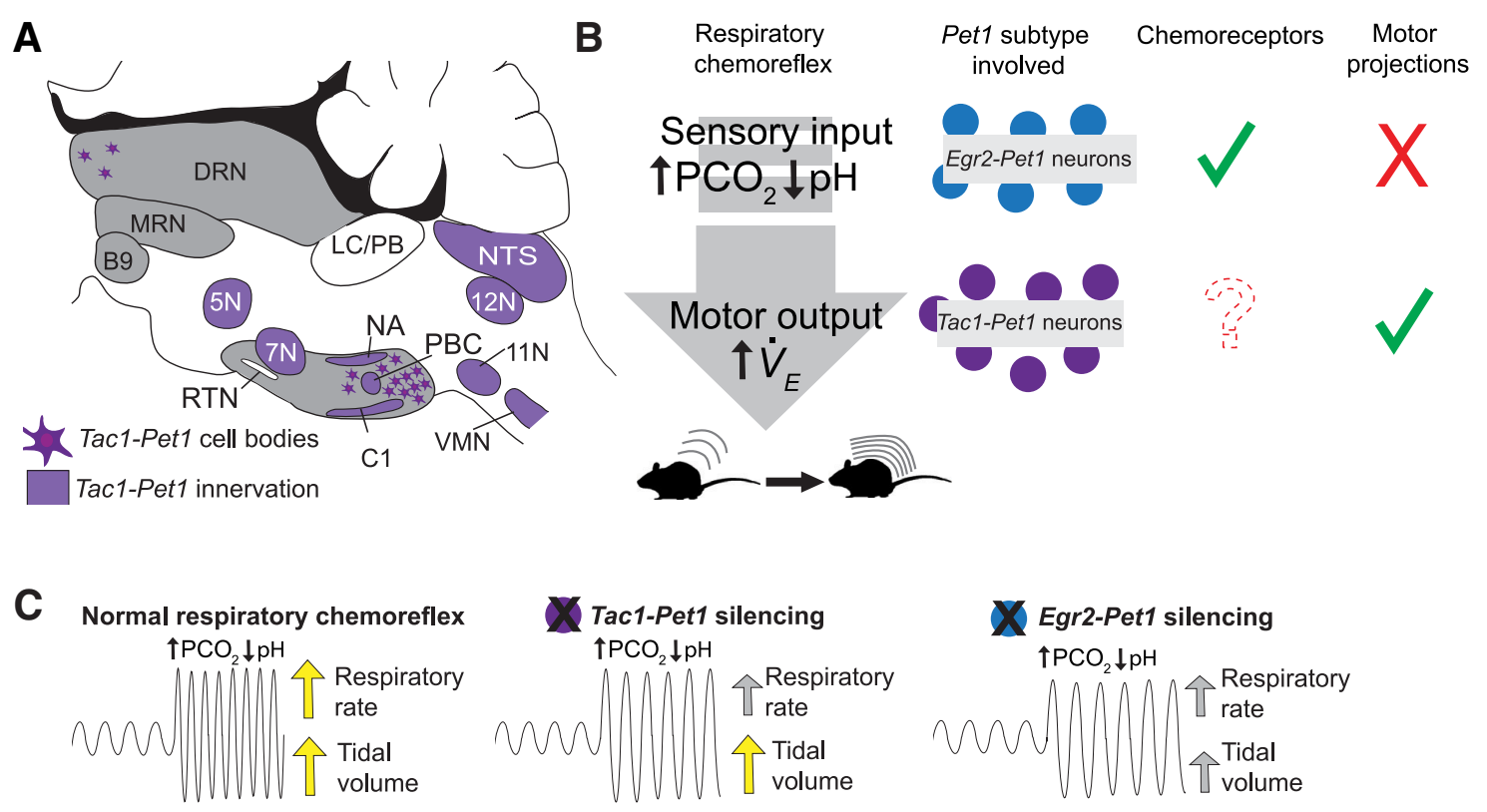

Figure 6. Summary of Tac1-Pet1 innervation, cell body locations, and Pet1 neuron subtypes in the respiratory chemoreflex. A, Schematic illustrating regions receiving Tac1-Pet1 projections throughout the brainstem and spinal cord and locations of cell bodies within the raphe nuclei. B, Contrasting roles of Egr2-Pet1 and Tac1-Pet1 neurons in respiratory chemoreflex. Egr2-Pet1 neurons have been shown to respond directly to decreased pH, whereas direct recordings of Tac1-Pet1 neurons have not yet been performed. Both Tac1-and Egr2-Pet1 neuron subsets project to chemosensory regions, whereas only Tac1-Pet1 neurons project to motor nuclei within the brainstem. C, Effect of silencing Egr2-Pet1 and Tac1-Pet1 subsets on the respiratory rate and tidal volume components of the respiratory chemoreflex.

(Iceman et al., 2013; Brust et al., 2014; Okaty et al., 2015), although this remains to be tested directly, as does probing Tac1Pet1 neurons within the paragigantocellularis lateralis. In sum, life-sustaining respiratory dynamics rely on brainstem centers including at least two distinct subtypes of Pet 1 raphe neurons: the r5Egr2-Pet1 neuron subtype involved in detection and processing of $\mathrm{CO}_{2}$ chemosensory input signals (Brust et al., 2014) and the Tac1-Pet1 subtype likely involved in the modulation of respiratory premotor and motor output during hypercapnic acidosis (Fig. 6).

\section{Distinct subtypes of Pet1 raphe neurons impinge on different components of the respiratory chemoreflex}

$\mathrm{CNO} / \mathrm{hM} 4 \mathrm{Di}$-triggered perturbation of Tac1-Pet1 neurons blunted the respiratory chemoreflex to $5 \% \mathrm{CO}_{2}$ by a percentage difference value of 42 percentage points, comparable to the effect found after perturbation of Egr2-Pet1 neurons (difference value of 36 percentage points) or all Pet1::Flpe-expressing neurons (difference value of 36 percentage points) (Brust et al., 2014). This suggests a model of serial action; perturbation of both subtypes simultaneously, as would occur upon silencing all Pet1::Flpe neurons, does not lead to a more severe respiratory phenotype than perturbing either subtype alone. This model is supported electrophysiologically and hodologically. r5Egr2-Pet1 neurons, raphe magnus constituents, respond directly to conditions of hypercapnic acidosis (Brust et al., 2014) and efferents restricted to brainstem chemosensory centers. In contrast, Tac1-Pet1 efferents densely innervate brainstem centers for respiratory rhythm generation, airway patency, and engagement of muscles of respiration. Moreover, indirect evidence suggests that Tac1-Pet1 neurons, especially those of the raphe obscurus, are likely not intrinsically chemosensitive (Brust et al., 2014). Supported, then, is a model in which r5Egr2-Pet1 neurons potentiate the respiratory chemoreflex via chemosensory input, whereas Tac1-Pet1 neurons do so via modulation of motor output and rhythm generation (Fig. 6). In addition, these two Pet1 neuron subtypes are likely separate cell lineages, occupying distinct raphe territories albeit with some intermingling. Whole-genome RNA sequencing of pooled and single Egr2-Pet1 neurons and raphe obscurus neurons show no overlap in Tacl expression; Tacl transcripts are undetectable in Egr2-Pet1 neurons and raphe obscurus Tac1 + Pet1 neurons do not express Egr2 (Okaty et al., 2015).

Potentiation of the respiratory chemoreflex by Tac1-Pet1 neurons stemmed from effects on respiratory rate; in contrast, disruption of Egr2-Pet1 neuron activity affected both rate and tidal volume (Brust et al., 2014; R. Brust, personal communication). The latter may reflect a more upstream role of chemosensation subserved by Egr2-Pet1 neurons, resulting in engagement of both pathways (frequency and volume) to increase ventilation in response to hypercapnia. Tac1-Pet1 neurons, though, may represent only one of the downstream effector groups impinging on respiratory chemoreflex output.

Resolution of 5-HT neuron subtype-specific functions helps to reconcile debates regarding 5-HT neurons in the respiratory chemoreflex. Reports arguing against a 5-HT neuron chemoreceptive function queried raphe obscurus neurons (Depuy et al., 2011), albeit under isoflurane anesthesia, which is known to inhibit 5-HT neuron activity (Massey et al., 2015). Our data also suggest that obscurus neurons are largely nonchemosensitive (Brust et al., 2014) and composed of Tac1-Pet1 neurons. Contrastingly, reports arguing for serotonergic neurons as intrinsic chemoreceptors (Veasey et al., 1995; Richerson, 2004; Hodges and Richerson, 2010; Ray et al., 2011; Iceman et al., 2013; Brust et al., 2014) queried what we now know as r5Egr2-Pet1 neurons, a subset indeed exhibiting intrinsic chemosensitivity (Brust et al., 2014). Both subtypes (Egr2-Pet1 and Tac1-Pet1) appear to be critical for the respiratory chemoreflex, but each likely influences different parts of the circuit: one perception of hypercapnic acidosis and transformation into respiratory drive and the other output to respiratory premotor and motor centers. 


\section{Innervation profile suggests a broader role for Tac1-Pet1 neurons in motor control}

Tac1-Pet1 boutons were found localized to somatic and autonomic motor regions in the brainstem, consistent with more general projection mapping data from raphe obscurus neurons (Loewy, 1981; Thor and Helke, 1987; Ellenberger et al., 1992; Depuy et al., 2011). Given this widespread innervation of motor targets, Tac1-Pet1 neurons may comprise part of the serotonergic circuitry that modulates basal motor tone (Jacobs and Fornal, 1991, 1997; Jacobs et al., 2002). Interestingly, Tac1-Pet1 neurons innervate face and neck muscles involved in rhythmic oral behaviors such as sucking, licking, and chewing, which require coordination of respiration to prevent aspiration of oral contents. In cats, these behaviors lead to an increase in firing rate among a population of dorsal raphe neurons, as well as obscurus and pallidus neurons (Veasey et al., 1995; Fornal et al., 1996). Therefore, Tac1-Pet1 neurons may be involved in the modulation of other rhythmic motor behaviors, for example, those requiring coordination with respiration.

Serotonergic neurons have been implicated in other respiratory reflexes, including long-term facilitation of respiration by repeated exposure to hypoxia. Stimulation of raphe obscurus neurons in anesthetized cats leads to long-term potentiation of phrenic nerve output (Millhorn, 1986), which is thought to represent the source of facilitation of respiration after either direct stimulation of carotid body afferents (Millhorn et al., 1980a, 1980b) or exposure to hypoxia (Olson et al., 2001). Further, depletion of spinal serotonin leads to an attenuation or even abolishment of long-term facilitation in anesthetized rats (BakerHerman and Mitchell, 2002). Given their location within the raphe obscurus and projections to spinal cord motor areas, Tac1Pet1 neurons may indeed be a source of serotonergic input modulating the generation of respiratory facilitation in response to hypoxia, a subject to pursue in future studies.

Although resident largely within the raphe obscurus and paragigantocellularis lateralis, Tac1-Pet1 neurons can be found to a small extent within raphe pallidus, a nucleus implicated in thermoregulation by way of projections to the spinal cord intermediolateral cell column harboring sympathetic output neurons that regulate shivering thermogenesis and brown fat metabolism (Hale et al., 2011; Morrison and Nakamura, 2011). Notably though, we observed no defect in thermoregulation upon $\mathrm{CNO} /$ hM4Di-mediated perturbation of Tac1-Pet1 neurons, contrasting the hypothermia phenotype induced upon suppression of Pet1::Flpe neurons en masse (Ray et al., 2011). These findings suggest that a separate group of serotonergic neurons within the pallidus modulates this thermogenesis circuit.

\section{Tac1-Pet1 neurons and sudden infant death syndrome}

Serotonergic abnormalities have been linked to several respiratory-related pathologies, including sudden infant death syndrome (SIDS) (Kinney et al., 2001; Kinney, 2009). Serotonin released at respiratory motor neurons appears to be important for respiratory plasticity in response to repeated apneic events (Baker-Herman and Mitchell, 2002), whereas 5-HT-deficient neonatal mice have failed heart rate recovery and excess mortality in response to hypoxia (Cummings et al., 2011a; Barrett et al., 2016). Furthermore, neonatal Tac1 ${ }^{-/-}$mice have defects in longterm respiratory facilitation in response to intermittent hypoxia and abnormal hypoxic respiratory responses (Berner et al., 2007), whereas substance P levels have been found to be elevated in SIDS cases, suggesting defects in both the serotonergic and substance P-producing systems in these infants (Bergström et al., 1984;
Obonai et al., 1996; Ozawa and Takashima, 2002). The question of whether Tac1-Pet1 neurons may play a role in respiratory regulation not only at adult time points, but also at early postnatal ages is an important one. We have found that Tac1-Pet1 neurons are present as early as P8 (data not shown) and future studies will be necessary to determine whether activity of these neurons is required for maintaining perinatal respiratory homeostasis.

In conclusion, this work confirms the importance of raphe obscurus and substance P-expressing Pet1 neurons in the control of respiration and for the first time aligns anatomical, molecular, functional, and projection data to describe a specialized subset of Tachykinin1-expressing Pet1 neurons with the ability to modulate breathing.

\section{References}

Andrade R, Haj-Dahmane S (2013) Serotonin neuron diversity in the dorsal raphe. ACS Chem Neurosci 4:22-25. CrossRef Medline

Armbruster BN, Li X, Pausch MH, Herlitze S, Roth BL (2007) Evolving the lock to fit the key to create a family of $\mathrm{G}$ protein-coupled receptors potently activated by an inert ligand. Proc Natl Acad Sci U S A 104:51635168. CrossRef Medline

Baker-Herman TL, Mitchell GS (2002) Phrenic long-term facilitation requires spinal serotonin receptor activation and protein synthesis. J Neurosci 22:6239-6246. Medline

Bang SJ, Jensen P, Dymecki SM, Commons KG (2012) Projections and interconnections of genetically defined serotonin neurons in mice. Eur J Neurosci 35:85-96. CrossRef Medline

Barrett KT, Dosumu-Johnson RT, Daubenspeck JA, Brust RD, Kreouzis V, Kim JC, Li A, Dymecki SM, Nattie EE (2016) Partial raphe dysfunction in neurotransmission is sufficient to increase mortality after anoxic exposures in mice at a critical period in postnatal development. J Neurosci 36:3943-3953. CrossRef Medline

Bergström L, Lagercrantz H, Terenius L (1984) Post-mortem analyses of neuropeptides in brains from sudden infant death victims. Brain Res 323:279-285. CrossRef Medline

Berner J, Shvarev Y, Lagercrantz H, Bilkei-Gorzo A, Hökfelt T, Wickström R (2007) Altered respiratory pattern and hypoxic response in transgenic newborn mice lacking the tachykinin-1 gene. J Appl Physiol 103:552-559. CrossRef Medline

Brust RD, Corcoran AE, Richerson GB, Nattie E, Dymecki SM (2014) Functional and developmental identification of a molecular subtype of brain serotonergic neuron specialized to regulate breathing dynamics. Cell Rep 9:2152-2165. CrossRef Medline

Cummings KJ, Commons KG, Hewitt JC, Daubenspeck JA, Li A, Kinney HC, Nattie EE (2011a) Failed heart rate recovery at a critical age in 5-HTdeficient mice exposed to episodic anoxia: implications for SIDS. J Appl Physiol 111:825-833. CrossRef Medline

Cummings KJ, Hewitt JC, Li A, Daubenspeck JA, Nattie EE (2011b) Postnatal loss of brainstem serotonin neurones compromises the ability of neonatal rats to survive episodic severe hypoxia. J Physiol 589:5247-5256. CrossRef Medline

Dean JB, Nattie EE (2010) Central CO2 chemoreception in cardiorespiratory control. J Appl Physiol 108:976-978. CrossRef Medline

Deneris ES (2011) Molecular genetics of mouse serotonin neurons across the lifespan. Neuroscience 197:17-27. CrossRef Medline

Depuy SD, Kanbar R, Coates MB, Stornetta RL, Guyenet PG (2011) Control of breathing by raphe obscurus serotonergic neurons in mice. J Neurosci 31:1981-1990. CrossRef Medline

Dias MB, Li A, Nattie E (2008) Focal CO2 dialysis in raphe obscurus does not stimulate ventilation but enhances the response to focal $\mathrm{CO} 2$ dialysis in the retrotrapezoid nucleus. J Appl Physiol 105:83-90. CrossRef Medline

Dymecki SM, Ray RS, Kim JC (2010) Mapping cell fate and function using recombinase-based intersectional strategies. Meth Enzymol 477:183-213. CrossRef Medline

Ellenberger HH, Vera PL, Feldman JL, Holets VR (1992) Multiple putative neuromessenger inputs to the phrenic nucleus in rat. Journal of Chemical Neuroanatomy 5:375-382. CrossRef Medline

Fernandez SP, Cauli B, Cabezas C, Muzerelle A, Poncer JC, Gaspar P (2016) Multiscale single-cell analysis reveals unique phenotypes of raphe 5-HT 
neurons projecting to the forebrain. Brain Struct Funct 221:4007-4025. Medline

Fornal CA, Metzler CW, Marrosu F, Ribiero-do-Valle LE, Jacobs BL (1996) A subgroup of dorsal raphe serotonergic neurons in the cat is strongly activated during oral-buccal movements. Brain Res 716:123-133. CrossRef Medline

Gaspar P, Lillesaar C (2012) Probing the diversity of serotonin neurons. Philos Trans R Soc Lond B Biol Sci 367:2382-2394. CrossRef Medline

Glazer EJ, Steinbusch H, Verhofstad A, Basbaum AI (1981) Serotonin neurons in nucleus raphe dorsalis and paragigantocellularis of the cat contain enkephalin. J Physiol (Paris) 77:241-245. Medline

Gray PA, Janczewski WA, Mellen N, McCrimmon DR, Feldman JL (2001) Normal breathing requires preBötzinger complex neurokinin-1 receptorexpressing neurons. Nat Neurosci 4:927-930. CrossRef Medline

Guyenet PG, Stornetta RL, Bayliss DA (2010) Central respiratory chemoreception. J Comp Neurol 518:3883-3906. CrossRef Medline

Hale MW, Lowry CA (2011) Functional topography of midbrain and pontine serotonergic systems: implications for synaptic regulation of serotonergic circuits. Psychopharmacology (Berl) 213:243-264. CrossRef Medline

Hale MW, Dady KF, Evans AK, Lowry CA (2011) Evidence for in vivo thermosensitivity of serotonergic neurons in the rat dorsal raphe nucleus and raphe pallidus nucleus implicated in thermoregulatory cooling. Exp Neurol 227:264-278. CrossRef Medline

Harris JA, Hirokawa KE, Sorensen SA, Gu H, Mills M, Ng LL, Bohn P, Mortrud M, Ouellette B, Kidney J, Smith KA, Dang C, Sunkin S, Bernard A, Oh SW, Madisen L, Zeng H (2014) Anatomical characterization of Cre driver mice for neural circuit mapping and manipulation. Front Neural Circuits 8:76. CrossRef Medline

Hodges MR, Richerson GB (2008) Contributions of 5-HT neurons to respiratory control: neuromodulatory and trophic effects. Respir Physiol Neurobiol 164:222-232. CrossRef Medline

Hodges MR, Richerson GB (2010) Medullary serotonin neurons and their roles in central respiratory chemoreception. Respir Physiol Neurobiol 173:256-263. CrossRef Medline

Hodges MR, Tattersall GJ, Harris MB, McEvoy SD, Richerson DN, Deneris ES, Johnson RL, Chen ZF, Richerson GB (2008) Defects in breathing and thermoregulation in mice with near-complete absence of central serotonin neurons. J Neurosci 28:2495-2505. CrossRef Medline

Holtman JR (1988) Immunohistochemical localization of serotonin- and substance $\mathrm{P}$-containing fibers around respiratory muscle motoneurons in the nucleus ambiguus of the cat. Neuroscience 26:169-178. CrossRef Medline

Hsiao CF, Wu N, Levine MS, Chandler SH (2002) Development and serotonergic modulation of NMDA bursting in rat trigeminal motoneurons. J Neurophysiol 87:1318-1328. Medline

Huckstepp RT, Cardoza KP, Henderson LE, Feldman JL (2015) Role of parafacial nuclei in control of breathing in adult rats. J Neurosci 35:10521067. CrossRef Medline

Iceman KE, Richerson GB, Harris MB (2013) Medullary serotonin neurons are CO2 sensitive in situ. J Neurophysiol 110:2536-2544. CrossRef Medline

Jacobs BL, Fornal CA (1991) Activity of brain serotonergic neurons in the behaving animal. Pharmacol Rev 43:563-578. Medline

Jacobs BL, Fornal CA (1997) Serotonin and motor activity. Curr Opin Neurobiol 7:820-825. CrossRef Medline

Jacobs BL, Martín-Cora FJ, Fornal CA (2002) Activity of medullary serotonergic neurons in freely moving animals. Brain Res Brain Res Rev 40:4552. CrossRef Medline

Jensen P, Farago AF, Awatramani RB, Scott MM, Deneris ES, Dymecki SM (2008) Redefining the serotonergic system by genetic lineage. Nat Neurosci 11:417-419. CrossRef Medline

Kachidian P, Poulat P, Marlier L, Privat A (1991) Immunohistochemical evidence for the coexistence of substance $\mathrm{P}$, thyrotropin-releasing hormone, GABA, methionine-enkephalin, and leucin-enkephalin in the serotonergic neurons of the caudal raphe nuclei: a dual labeling in the rat. J Neurosci Res 30:521-530. CrossRef Medline

Kim JC, Cook MN, Carey MR, Shen C, Regehr WG, Dymecki SM (2009) Linking genetically defined neurons to behavior through a broadly applicable silencing allele. Neuron 63:305-315. CrossRef Medline

Kinney HC (2009) Brainstem mechanisms underlying the sudden infant death syndrome: evidence from human pathologic studies. Dev Psychobiol 51:223-233. CrossRef Medline

Kinney HC, Filiano JJ, White WF (2001) Medullary serotonergic network deficiency in the sudden infant death syndrome: review of a 15-year study of a single dataset. J Neuropathol Exp Neurol 60:228-247. CrossRef Medline

Li L, Tasic B, Micheva KD, Ivanov VM, Spletter ML, Smith SJ, Luo L (2010) Visualizing the distribution of synapses from individual neurons in the mouse brain. PLoS One 5:e11503. CrossRef Medline

Loewy AD (1981) Raphe pallidus and raphe obscurus projections to the intermediolateral cell column in the rat. Brain Res 222:129-133. CrossRef Medline

Massey CA, Iceman KE, Johansen SL, Wu Y, Harris MB, Richerson GB (2015) Isoflurane abolishes spontaneous firing of serotonin neurons and masks their pH/CO 2chemosensitivity. J Neurophysiol 113:2879-2888. CrossRef Medline

Millhorn DE (1986) Stimulation of raphe (obscurus) nucleus causes longterm potentiation of phrenic nerve activity in cat. J Physiol 381:169-179. CrossRef Medline

Millhorn DE, Eldridge FL, Waldrop TG (1980a) Prolonged stimulation of respiration by endogenous central serotonin. Respir Physiol 42:171-188. CrossRef Medline

Millhorn DE, Eldridge FL, Waldrop TG (1980b) Prolonged stimulation of respiration by a new central neural mechanism. Respir Physiol 41:87-103. CrossRef Medline

Morrison SF, Nakamura K (2011) Central neural pathways for thermoregulation. Front Biosci (Landmark Ed) 16:74-104. CrossRef Medline

Mulkey DK, Hawkins VE, Hawryluk JM, Takakura AC, Moreira TS, Tzingounis AV (2015) Molecular underpinnings of ventral surface chemoreceptor function: focus on KCNQ channels. J Physiol 593:1075-1081. CrossRef Medline

Nakamura M, Yasuda K, Hasumi-Nakayama Y, Sugiura M, Tomita I, Mori R, Tanaka S, Furusawa K (2006) Colocalization of serotonin and substance $\mathrm{P}$ in the postnatal rat trigeminal motor nucleus and its surroundings. Int J Dev Neurosci 24:61-64. CrossRef Medline

Nakamura A, Zhang W, Yanagisawa M, Fukuda Y, Kuwaki T (2007) Vigilance state-dependent attenuation of hypercapnic chemoreflex and exaggerated sleep apnea in orexin knockout mice. J Appl Physiol 102:241-248. CrossRef Medline

Nakata T, Terada S, Hirokawa N (1998) Visualization of the dynamics of synaptic vesicle and plasma membrane proteins in living axons. J Cell Biol 140:659-674. CrossRef Medline

Nattie E, Li A (2006) Neurokinin-1 receptor-expressing neurons in the ventral medulla are essential for normal central and peripheral chemoreception in the conscious rat. J Appl Physiol 101:1596-1606. CrossRef Medline

Nattie E, Li A (2012) Central chemoreceptors: locations and functions. Compr Physiol 2:221-254. CrossRef Medline

Niederkofler V, Asher TE, Okaty BW, Rood BD, Narayan A, Hwa LS, Beck SG, Miczek KA, Dymecki SM (2016) Identification of serotonergic neuronal modules that affect aggressive behavior. Cell Rep 17:1934-1949. CrossRef Medline

Nuding SC, Segers LS, Shannon R, O'Connor R, Morris KF, Lindsey BG (2009) Central and peripheral chemoreceptors evoke distinct responses in simultaneously recorded neurons of the raphé-pontomedullary respiratory network. Philos Trans R Soc Lond B Biol Sci 364:2501-2516. CrossRef Medline

Obonai T, Takashima S, Becker LE, Asanuma M, Mizuta R, Horie H, Tanaka J (1996) Relationship of substance P and gliosis in medulla oblongata in neonatal sudden infant death syndrome. Pediatr Neurol 15:189-192. CrossRef Medline

Okaty BW, Freret ME, Rood BD, Brust RD, Hennessy ML, deBairos D, Kim JC, Cook MN, Dymecki SM (2015) Multi-scale molecular deconstruction of the serotonin neuron system. Neuron 88:774-791. CrossRef Medline

Olson EB Jr, Bohne CJ, Dwinell MR, Podolsky A, Vidruk EH, Fuller DD, Powell FL, Mitchel GS (2001) Ventilatory long-term facilitation in unanesthetized rats. J Appl Physiol (1985) 91:709-716. Medline

Ozawa Y, Takashima S (2002) Developmental neurotransmitter pathology in the brainstem of sudden infant death syndrome: a review and sleep position. Forensic Sci Int 130:S53-S59. Medline

Peever JH, Necakov A, Duffin J (2001) Nucleus raphé obscurus modulates 
hypoglossal output of neonatal rat in vitro transverse brain stem slices. J Appl Physiol (1985) 90:269-279. Medline

Pennuto M, Dunlap D, Contestabile A, Benfenati F, Valtorta F (2002) Fluorescence resonance energy transfer detection of synaptophysin I and vesicle-associated membrane protein 2 interactions during exocytosis from single live synapses. Mol Biol Cell 13:2706-2717. CrossRef Medline

Pilowsky PM (2014) Peptides, serotonin, and breathing: the role of the raphe in the control of respiration. Prog Brain Res 209:169-189. CrossRef Medline

Ptak K, Yamanishi T, Aungst J, Milescu LS, Zhang R, Richerson GB, Smith JC (2009) Raphe neurons stimulate respiratory circuit activity by multiple mechanisms via endogenously released serotonin and substance P. J Neurosci 29:3720-3737. CrossRef Medline

Ray RS, Corcoran AE, Brust RD, Kim JC, Richerson GB, Nattie E, Dymecki SM (2011) Impaired respiratory and body temperature control upon acute serotonergic neuron inhibition. Science 333:637-642. CrossRef Medline

Richerson GB (2004) Serotonergic neurons as carbon dioxide sensors that maintain $\mathrm{pH}$ homeostasis. Nat Rev Neurosci 5:449-461. CrossRef Medline

Saboisky JP, Butler JE, Fogel RB, Taylor JL, Trinder JA, White DP, Gandevia SC (2006) Tonic and phasic respiratory drives to human genioglossus motoneurons during breathing. J Neurophysiol 95:2213-2221. Medline

Spaethling JM, Piel D, Dueck H, Buckley PT, Morris JF, Fisher SA, Lee J, Sul JY, Kim J, Bartfai T, Beck SG, Eberwine JH (2014) Serotonergic neuron regulation informed by in vivo single-cell transcriptomics. FASEB J 28: 771-780. CrossRef Medline

Stachniak TJ, Ghosh A, Sternson SM (2014) Chemogenetic synaptic silencing of neural circuits localizes a hypothalamus $\rightarrow$ midbrain pathway for feeding behavior. Neuron 82:797-808. CrossRef Medline
Stamp JA, Semba K (1995) Extent of colocalization of serotonin and GABA in the neurons of the rat raphe nuclei. Brain Res 677:39-49. CrossRef Medline

Stornetta RL, Moreira TS, Takakura AC, Kang BJ, Chang DA, West GH, Brunet JF, Mulkey DK, Bayliss DA, Guyenet PG (2006) Expression of Phox $2 \mathrm{~b}$ by brainstem neurons involved in chemosensory integration in the adult rat. J Neurosci 26:10305-10314. CrossRef Medline

Tallaksen-Greene SJ, Elde R, Wessendorf MW (1993) Regional distribution of serotonin and substance $\mathrm{P}$ co-existing in nerve fibers and terminals in the brainstem of the rat. Neuroscience 53:1127-1142. CrossRef Medline

Teissier A, Chemiakine A, Inbar B, Bagchi S, Ray RS, Palmiter RD, Dymecki SM, Moore H, Ansorge MS (2015) Activity of raphé serotonergic neurons controls emotional behaviors. Cell Rep 13:1965-1976. CrossRef Medline

Thor KB, Helke CJ (1987) Serotonin- and substance P-containing projections to the nucleus tractus solitarii of the rat. J Comp Neurol 265:275293. CrossRef Medline

Veasey SC, Fornal CA, Metzler CW, Jacobs BL (1995) Response of serotonergic caudal raphe neurons in relation to specific motor activities in freely moving cats. J Neurosci 15:5346-5359. Medline

Wang F, Flanagan J, Su N, Wang LC, Bui S, Nielson A, Wu X, Vo HT, Ma XJ, Luo Y (2012) RNAscope: a novel in situ RNA analysis platform for formalin-fixed, paraffin-embedded tissues. J Mol Diagn 14:22-29. CrossRef Medline

Wang W, Tiwari JK, Bradley SR, Zaykin RV, Richerson GB (2001) Acidosisstimulated neurons of the medullary raphe are serotonergic. J Neurophysiol 85:2224-2235. Medline

Zhang C, Yan H, Li C, Zheng Y (2004) Possible involvement of the facial nucleus in regulation of respiration in rats. Neurosci Lett 367:283-288. CrossRef Medline 